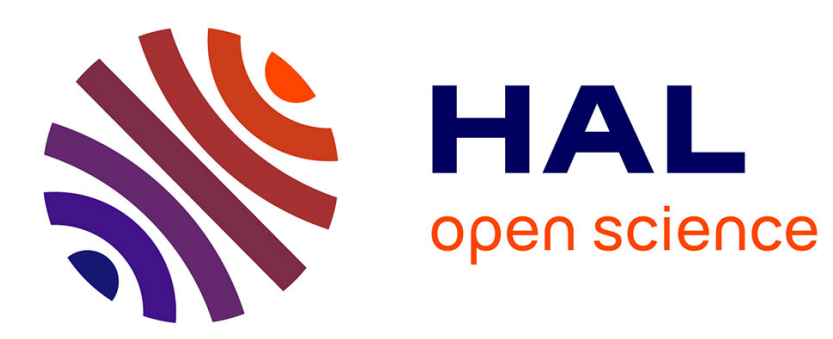

\title{
Kinematic Analysis of the 3-RPS-3-SPR Series-Parallel Manipulator
}

\author{
Abhilash Nayak, Stéphane Caro, Philippe Wenger
}

\section{To cite this version:}

Abhilash Nayak, Stéphane Caro, Philippe Wenger. Kinematic Analysis of the 3-RPS-3-SPR SeriesParallel Manipulator. Robotica, 2019, 37 (7), pp.1240-1266. 10.1017/S0263574718000826 . hal01859984

\section{HAL Id: hal-01859984 https://hal.science/hal-01859984}

Submitted on 22 Aug 2018

HAL is a multi-disciplinary open access archive for the deposit and dissemination of scientific research documents, whether they are published or not. The documents may come from teaching and research institutions in France or abroad, or from public or private research centers.
L'archive ouverte pluridisciplinaire HAL, est destinée au dépôt et à la diffusion de documents scientifiques de niveau recherche, publiés ou non, émanant des établissements d'enseignement et de recherche français ou étrangers, des laboratoires publics ou privés. 


\title{
Kinematic Analysis of the 3-RPS-3-SPR Series-Parallel Manipulator
}

\author{
Abhilash Nayak†, Stéphane Caro§, and Philippe Wenger§ \\ †École Centrale de Nantes, \\ $\S$ Centre National de Recherche Scientifique (CNRS), \\ Laboratoire des Sciences du Numérique de Nantes (LS2N), \\ UMR CNRS 6004
}

\begin{abstract}
This paper deals with the kinematic analysis and enumeration of singularities of the six degree-of-freedom 3-RPS-3-SPR series-parallel manipulator. The characteristic tetrahedron of the S-PM is established, whose degeneracy is bijectively mapped to the serial singularities of the S-PM. Study parametrization is used to determine six independent parameters that characterize the S-PM and the direct kinematics problem is solved by mapping the transformation matrix between the base and the end-effector to a point in $\mathbb{P}^{7}$. The inverse kinematics problem of the 3-RPS-3-SPR series-parallel manipulator amounts to find the location of three points on three lines. This problem leads to a minimal octic univariate polynomial with four quadratic factors.

Keywords: Series-parallel manipualtors; S-PM; Singularities; characteristic tetrahedron; inverse kinematics; direct kinematics
\end{abstract}

\section{Introduction}

Serial and parallel manipulators (SM and PM) have received a lot of interest for the last few decades due to the high stiffness properties of parallel manipulators ${ }^{1}$ and large workspace of serial manipulators. ${ }^{2}$ Hence a marriage between serial and parallel manipulators with a hope to reap the merits of

\footnotetext{
${ }^{*}$ Corresponding author. E-mail: stephane.caro@ls2n.fr
} 
both, has led to hybrid manipulators. ${ }^{3-9}$ A hybrid manipulator is a serial linkage mounted on a parallel manipulator and vice-versa or a serial arrangement of two or more parallel manipulators, known as a Series-Parallel Manipulator (S-PM). As much as it is regarded for its merits, a S-PM also bears the demerits of its constituent manipulators in the sense that its kinematic modeling and singularity analysis are more complicated. Various approaches have been proposed in the literature to analyze S-PMs: Shahinpoor ${ }^{5}$ solved the direct and inverse kinematics of modular 3 -axis parallel manipulators mounted in series as an $n$-axis S-PM. Romdhane ${ }^{8}$ performed the forward displacement analysis of a Stewart-like S-PM. Tanev ${ }^{3}$ studied a novel 6 degrees of freedom (dof) S-PM and derived the closed-form solutions to its forward and inverse kinematics. Moreover, Zheng et al. ${ }^{9}$ obtained closed-form kinematic solutions for the design of a 6-dof S-PM composed of a 3-UPU translational PM and a 3-UPU rotational PM mounted in series. In most of these S-PMs, the constituent modules possess the degrees of freedom that are pure rotations or translations. Hence, each module can be replaced by a set of equivalent lower kinematic pairs that can simplify the understanding of the S-PM behaviour. There exist other S-PMs in which the PMs that constitute them have their degrees of freedom coupled and hence give rise to parasitic motions. $\mathrm{Hu}$, $\mathrm{Lu}$ and Alvarado ${ }^{10-12,14,15}$ have contributed considerably to the design and analysis of this kind of series-parallel manipulators. $\mathrm{Lu}$ and $\mathrm{Hu}^{11}$ pursued the kinematic analysis of a $2(\mathrm{SP}+\mathrm{SPR}+\mathrm{SPU}) \mathrm{S}-\mathrm{PM}$ and plotted its workspace. They also performed the static analysis ${ }^{10}$ of S-PMs with $k$-PMs in series. In addition, $\mathrm{Hu}^{14}$ formulated the Jacobian matrix for S-PMs as a function of Jacobians of the individual parallel modules. Alvarado ${ }^{12}$ used screw theory and the principle of virtual work to carry out the kinematic and dynamic analysis of a 2-(3-RPS) S-PM. The 3-RPS-3-SPR S-PM is another example of a S-PM composed of two parallel modules with coupled degrees of freedom. The proximal module is the 3-RPS parallel mechanism which performs a translation and two non pure rotations about non fixed axes, which induce two translational parasitic motions ${ }^{16}$ while the distal module is the 3 -SPR PM that has the same type of $d o f .{ }^{24} \mathrm{Hu}$ et al. ${ }^{13}$ analyzed the workspace of this manipulator. Alvarado et al. ${ }^{15}$ erroneously claimed that this S-PM has 5 dof. The reader is referred to as Nayak et al. ${ }^{17}$ for a better understanding of the mobility of this S-PM. Nayak et al. ${ }^{17}$ proved that the full-cycle mobility of this manipulator is equal to six. Nonetheless, there is very little research on the singularities of S-PMs. It is known that if any of the parallel modules are in a singular configuration, the S-PM is also singular ${ }^{3}$ but the singularities that arise due to the serial arrangement of the PMs are generally left out. This paper focuses on the enumeration of those serial singularities in the 3-RPS-3-SPR S-PM. It is shown that six independent parameters can 
be used to describe the kinematics of this manipulator. Furthermore, direct and inverse kinematics problems for the 3-RPS-3-SPR S-PM are solved using Study parametrization.

The paper is organized as follows: The manipulator under study is described in Section 2. Six independent parameters that characterize the S-PM are determined in Section 3. Section 4 presents the singularities of the S-PM, while pointing out its serial singular configurations. Section 5 and 6 deal with the direct and inverse kinematics problems of the 3-RPS-3-SPR S-PM.

\section{Architecture of the 3-RPS-3-SPR series par- allel manipulator}

The architecture of the 3-RPS-3-SPR S-PM under study is shown in Fig. 1. It consists of a proximal 3-RPS PM module and a distal 3-SPR PM module. The 3-RPS PM is composed of three legs each containing a revolute, a prismatic and a spherical joint mounted in series, while the legs of the 3-SPR PM have these lower pairs in reverse order. Thus, the three equilateral triangular shaped platforms are the fixed base, the coupler and the end effector, coloured brown, green and gray, respectively. The vertices of these platforms are named $A_{i}, B_{i}$ and $C_{i}, i=1,2,3$, respectively. Hereafter, the subscript 0 corresponds to the fixed base, 1 to the coupler platform and 2 to the endeffector. A coordinate frame $\mathcal{F}_{i}$ is attached to each platform such that its origin $O_{i}$ lies at its circumcenter. The coordinate axes, $x_{i}$ points towards the vertex $P_{1}, P=A, B, C y_{i}$ is parallel to the opposite side $P_{3} P_{2}$ and by the right hand rule, $z_{i}$ is normal to platform plane. Besides, the circum-radius of the $i$-th platform is denoted as $h_{i}$. $\mathbf{p}_{i}$ and $\mathbf{q}_{i}, i=1, \ldots, 6$ are unit vectors along the prismatic joints while $\mathbf{u}_{i}$ and $\mathbf{v}_{i}, i=1, \ldots, 6$ are unit vectors along the revolute joint axes. $\alpha_{i}$ is the plane passing through $A_{i}$ with its normal along $\mathbf{u}_{i}$. Similarly, $\beta_{i}$ is the plane passing through $C_{i}$ with its normal along $\mathbf{v}_{i}$. The spherical joint center $B_{i}$ is constrained to lie in planes $\alpha_{i}$ and $\beta_{i}$ simultaneously.

\section{Parametric representation of the 3-RPS-3- SPR series-parallel manipulator}

This section describes the parametrization of the 3-RPS-3-SPR series-parallel manipulator shown in Fig. 1. It will be shown that six independent parameters are sufficient to describe the position and orientation of the moving 


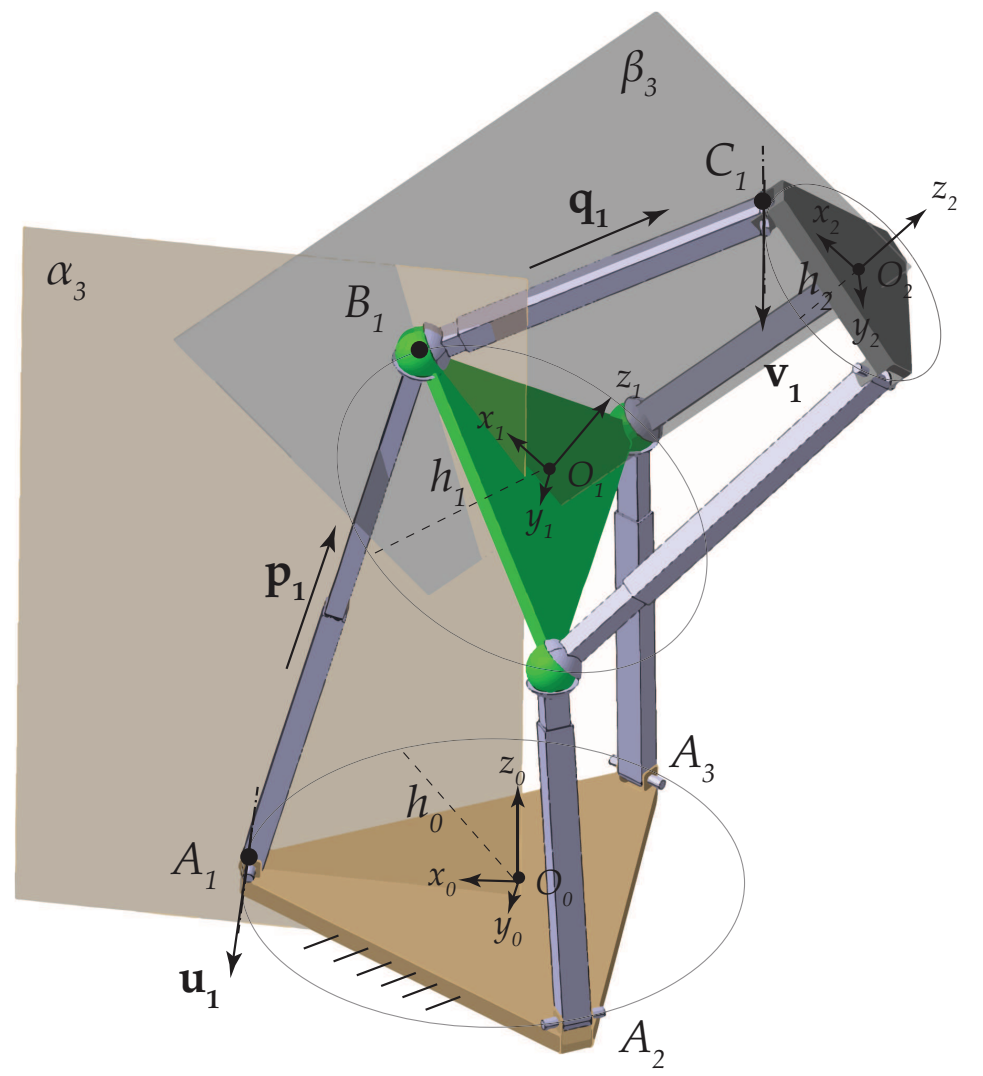

Figure 1: A 3-RPS-3-SPR series-parallel manipulator 
platform. These parameters are obtained by individually parametrizing the proximal and the distal modules.

Study's kinematic mapping ${ }^{18}$ maps each spatial Euclidean displacement $\gamma$ of $\mathrm{SE}(3)$ onto a point in 7-dimensional projective space, $\mathbf{p} \in \mathbb{P}^{7}$. In this parametrization, a point $[x, y, z]$ is transformed to $\left[x^{\prime}, y^{\prime}, z^{\prime}\right]$ according to:

$$
\left[1, x^{\prime}, y^{\prime}, z^{\prime}\right]^{T}=\mathbf{M}[1, x, y, z]^{T}
$$

where, the matrix $\mathbf{M} \in S E(3)$ is represented as:

$$
\begin{aligned}
& \mathbf{M}=\left[\begin{array}{cc}
x_{0}{ }^{2}+x_{1}{ }^{2}+x_{2}{ }^{2}+x_{3}{ }^{2} & \mathbf{0}_{3 \times 1}^{T} \\
\mathbf{M}_{T} & \mathbf{M}_{R}
\end{array}\right] \\
& \mathbf{M}_{T}=\left[\begin{array}{l}
-2 x_{0} y_{1}+2 x_{1} y_{0}-2 x_{2} y_{3}+2 x_{3} y_{2} \\
-2 x_{0} y_{2}+2 x_{1} y_{3}+2 x_{2} y_{0}-2 x_{3} y_{1} \\
-2 x_{0} y_{3}-2 x_{1} y_{2}+2 x_{2} y_{1}+2 x_{3} y_{0}
\end{array}\right] \\
& \mathbf{M}_{R}=\left[\begin{array}{ccc}
x_{0}{ }^{2}+x_{1}{ }^{2}-x_{2}{ }^{2}-x_{3}{ }^{2} & -2 x_{0} x_{3}+2 x_{1} x_{2} & 2 x_{0} x_{2}+2 x_{1} x_{3} \\
2 x_{0} x_{3}+2 x_{1} x_{2} & x_{0}{ }^{2}-x_{1}{ }^{2}+x_{2}{ }^{2}-x_{3}{ }^{2} & -2 x_{0} x_{1}+2 x_{3} x_{2} \\
-2 x_{0} x_{2}+2 x_{1} x_{3} & 2 x_{0} x_{1}+2 x_{3} x_{2} & x_{0}{ }^{2}-x_{1}{ }^{2}-x_{2}{ }^{2}+x_{3}{ }^{2}
\end{array}\right]
\end{aligned}
$$

where $\mathbf{M}_{T}$ and $\mathbf{M}_{R}$ represent the translational and rotational parts of the transformation matrix $\mathbf{M}$ respectively. The parameters $x_{i}, y_{i}, i \in\{0, \ldots, 3\}$ present in the transformation matrix $\mathbf{M}$ are called the Study-parameters. An Euclidean transformation can be represented by a point $\mathbf{p} \in \mathbb{P}^{7}$ if and only if the following equation and inequality are satisfied:

$$
\begin{array}{r}
x_{0} y_{0}+x_{1} y_{1}+x_{2} y_{2}+x_{3} y_{3}=0 \\
x_{0}{ }^{2}+x_{1}{ }^{2}+x_{2}{ }^{2}+x_{3}{ }^{2} \neq 0
\end{array}
$$

All the points that satisfy the Equation (5) belong to the 6-dimensional Study quadric, $S_{6}^{2}$. To avoid the inequality (6), usually a normalization condition $x_{i}=1, i=0,1,2$ or 3 or $x_{0}^{2}+x_{1}^{2}+x_{2}^{2}+x_{3}^{2}=1$ is used.

A geometric constraint for each leg of the 3-RPS parallel manipulator is that the spherical joint center is restricted to move in the plane whose normal is directed along the revolute joint axis. Let $f_{0}, f_{1}, f_{2}, f_{3}, g_{0}, g_{1}, g_{2}, g_{3}$ be the Study parameters. Using Study's kinematic mapping, ${ }^{18,19}$ three plane constraint equations $E_{i}=0, i=1,2,3$ can be written as a function of Study parameters $f_{i}, g_{i}, i=0,1,2,3$. Along with the Study's quadric $E_{4}$, there are four constraint equations irrespective of the actuation scheme:

$$
E_{1}:=f_{0} f_{3}=0
$$




$$
\begin{aligned}
& E_{2}:=f_{1}^{2} h_{1}-h_{1} f_{2}^{2}+2 f_{0} g_{1}-2 f_{1} g_{0}-2 f_{3} g_{2}+2 g_{3} f_{2}=0 \\
& E_{3}:=-2 f_{0} f_{3} h_{1}+f_{1} f_{2} h_{1}-f_{0} g_{2}+f_{1} g_{3}+f_{2} g_{0}-f_{3} g_{1}=0 \\
& E_{4}:=f_{0} g_{0}+f_{1} g_{1}+f_{2} g_{2}+f_{3} g_{3}=0
\end{aligned}
$$

where, $h_{1}$ is the circum-radius of the coupler platform. A different set of Study parameters, $\left[c_{0}, c_{1}, c_{2}, c_{3}, d_{0}, d_{1}, d_{2}, d_{3}\right]$ are considered to parameterize the distal module to distinguish the two modules. The constraint equations for the 3-SPR PM can be obtained by considering the conjugate of the dual quaternion of the 3 -RPS PM. ${ }^{20}$ In other words, assigning

$$
f_{0}=c_{0}, f_{1}=-c_{1}, f_{2}=-c_{2}, f_{3}=-c_{3}, g_{0}=d_{0}, g_{1}=-d_{1}, g_{2}=-d_{2}, g_{3}=-d_{3}
$$

in Eq. (7) to (10) yields the necessary equations.

Each module is a three dof parallel manipulator and to express these mobilities in terms of three parameters, the mechanism should be considered in one of its operation modes. For example, for the 3 -RPS module, $f_{3}=0$ represents one of its two operation modes. ${ }^{21}$ In this operation mode, $f_{0}$ can never be zero. This fact can be exploited to avoid any point $\left[f_{0}, f_{1}, f_{2}, f_{3}, g_{0}, g_{1}, g_{2}, g_{3}\right]$ of $\mathbb{P}^{7}$ to lie on the exceptional generator $f_{0}=f_{1}=f_{2}=f_{3}=0$. This is done by using the normalizing condition, $f_{0}=1$. By substituting $f_{3}=0$ and $f_{0}=1$ in Eqs. (8) to (10), $g_{0}, g_{2}$ and $g_{3}$ can be linearly solved as follows:

$$
\begin{aligned}
& g_{0}=1 / 2 \frac{f_{1} h_{1}\left(f_{1}{ }^{2}-3 f_{2}{ }^{2}\right)}{f_{1}{ }^{2}+f_{2}{ }^{2}+1} \\
& g_{2}=-1 / 2 \frac{f_{1}\left(2 f_{1}{ }^{2} g_{1}+f_{1}{ }^{2} h_{1}+2{f_{2}}^{2} g_{1}-3 f_{2}{ }^{2} h_{1}+2 g_{1}\right)}{f_{2}\left(f_{1}{ }^{2}+f_{2}{ }^{2}+1\right)} \\
& g_{3}=-1 / 2 \frac{3{f_{1}}^{2}{f_{2}}^{2} h_{1}-f_{2}{ }^{4} h_{1}+2{f_{1}}^{2} g_{1}+f_{1}{ }^{2} h_{1}+2{f_{2}}^{2} g_{1}-{f_{2}}^{2} h_{1}+2 g_{1}}{f_{2}\left(f_{1}{ }^{2}+f_{2}{ }^{2}+1\right)}
\end{aligned}
$$

Thus, the Euclidean transformation matrix for the proximal module in its operation mode $f_{3}=0$ can be written as a function of only three parameters $f_{1}, f_{2}$ and $g_{1}$ :

$$
\mathbf{T}_{1}=\left[\begin{array}{cccc}
1 & 0 & 0 & 0 \\
\frac{h_{1}\left(f_{1}{ }^{2}-f_{2}{ }^{2}\right)}{f_{1}{ }^{2}+f_{2}{ }^{2}+1} & \frac{f_{1}{ }^{2}-f_{2}{ }^{2}+1}{f_{1}{ }^{2}+f_{2}{ }^{2}+1} & \frac{2 f_{1} f_{2}}{f_{1}{ }^{2}+f_{2}{ }^{2}+1} & \frac{2 f_{2}}{f_{1}{ }^{2}+f_{2}{ }^{2}+1} \\
-\frac{2 h_{1} f_{2} f_{1}}{f_{1}{ }^{2}+f_{2}{ }^{2}+1} & \frac{2 f_{1} f_{2}}{f_{1}{ }^{2}+f_{2}{ }^{2}+1} & -\frac{f_{1}{ }^{2}-f_{2}{ }^{2}-1}{f_{1}{ }^{2}+f_{2}{ }^{2}+1} & -\frac{2 f_{1}}{f_{1}{ }^{2}+f_{2}{ }^{2}+1} \\
\frac{2 f_{1}{ }^{2} g_{1}+f_{1} h_{1}+2 f_{2}{ }^{2} g_{1}-f_{2}{ }^{2} h_{1}+2 g_{1}}{f_{2}\left(f_{1}{ }^{2}+f_{2}{ }^{2}+1\right)} & -\frac{2 f_{2}}{f_{1}{ }^{2}+f_{2}{ }^{2}+1} & \frac{2 f_{1}}{f_{1}{ }^{2}+f_{2}{ }^{2}+1} & -\frac{f_{1}{ }^{2}+f_{2}{ }^{2}-1}{f_{1}{ }^{2}+f_{2}{ }^{2}+1}
\end{array}\right]
$$

Similarly for the distal 3-SPR module in its operation mode corresponding to $c_{3}=0$, normalizing $c_{0}=1$ and eliminating $d_{0}, d_{2}$ and $d_{3}$, the transformation 
matrix can be derived as follows:

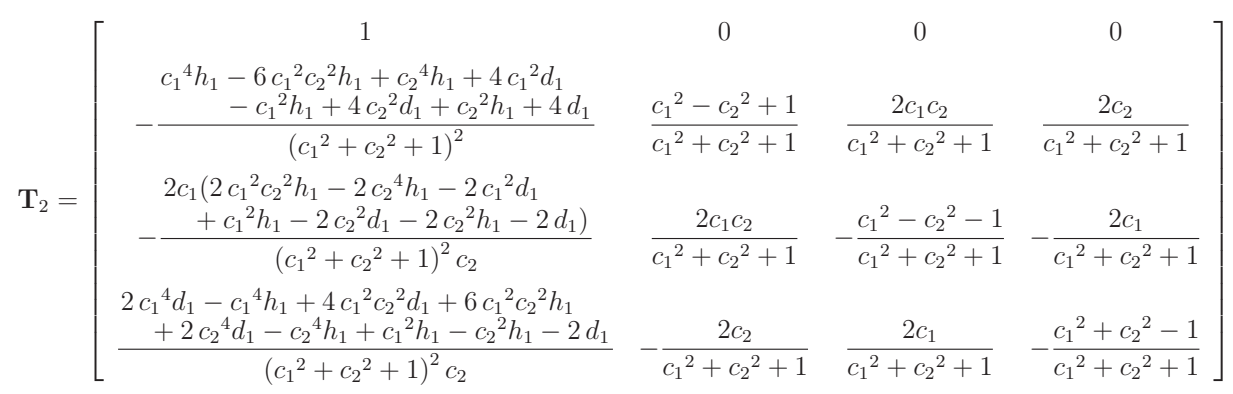

Therefore, a transformation matrix between the base frame $\mathcal{F}_{0}$ and the moving frame $\mathcal{F}_{2}$ can be expressed as $\mathbf{T}=\mathbf{T}_{1} \cdot \mathbf{T}_{2}$ and is a function of six independent parameters. For instance, when both the modules are in the operation mode represented by $f_{3}=0$ and $c_{3}=0, \mathbf{T}$ is a function of $f_{1}, f_{2}, g_{1}, c_{1}, c_{2}$ and $d_{1}$ and as a result, indicates that it is indeed a six dof mechanism. All possible configurations of the S-PM with its individual modules in different operation modes, include the following cases:

$$
\begin{array}{lll}
\text { Case } & \text { a. } & c_{3}=f_{3}=0 \\
\text { Case } & \text { b. } & c_{0}=f_{0}=0 \\
\text { Case } & \text { c. } & c_{0}=f_{3}=0 \\
\text { Case } & \text { d. } & c_{3}=f_{0}=0
\end{array}
$$

It will be shown in the subsequent sections how simple it is to adapt the results of Case a. to the remaining three cases.

Consequently, matrices $\mathbf{T}_{1}, \mathbf{T}_{2}$ and $\mathbf{T}$ can be used to express the coordinates of all the vectors in one frame, preferably the fixed co-ordinate frame $\mathcal{F}_{0}$ as follows:

$$
\begin{aligned}
&{ }^{0} \mathbf{r}_{A_{1}}=\left[1, h_{0}, 0,0\right]^{T} ; \quad{ }^{0} \mathbf{r}_{A_{2}}=\left[1,-\frac{h_{0}}{2}, \frac{\sqrt{3} h_{0}}{2}, 0\right]^{T} ; \quad{ }^{0} \mathbf{r}_{A_{3}}=\left[1,-\frac{h_{0}}{2},-\frac{\sqrt{3} h_{0}}{2}, 0\right]^{T} \\
&{ }^{1} \mathbf{r}_{B_{1}}=\left[1, h_{1}, 0,0\right]^{T} ; \quad{ }^{1} \mathbf{r}_{B_{2}}=\left[1,-\frac{h_{1}}{2}, \frac{\sqrt{3} h_{1}}{2}, 0\right]^{T} ; \quad{ }^{1} \mathbf{r}_{B_{3}}=\left[1,-\frac{h_{1}}{2},-\frac{\sqrt{3} h_{1}}{2}, 0\right]^{T} \\
&{ }^{2} \mathbf{r}_{C_{1}}=\left[1, h_{2}, 0,0\right]^{T} ; \quad{ }^{2} \mathbf{r}_{C_{2}}=\left[1,-\frac{h_{2}}{2}, \frac{\sqrt{3} h_{2}}{2}, 0\right]^{T} ; \quad{ }^{2} \mathbf{r}_{C_{3}}=\left[1,-\frac{h_{2}}{2},-\frac{\sqrt{3} h_{2}}{2}, 0\right]^{T} \\
&{ }^{0} \mathbf{u}_{1}==[0,0,1,0]^{T} ; \quad{ }^{0} \mathbf{u}_{2}=\left[1,-\frac{\sqrt{3}}{2},-\frac{1}{2}, 0\right]^{T} ; \quad{ }^{0} \mathbf{u}_{3}=\left[1, \frac{\sqrt{3}}{2},-\frac{1}{2}, 0\right]^{T} \\
&{ }^{2} \mathbf{v}_{1}==[0,0,1,0]^{T} ; \quad{ }^{2} \mathbf{v}_{2}=\left[1,-\frac{\sqrt{3}}{2},-\frac{1}{2}, 0\right]^{T} ; \quad{ }^{2} \mathbf{v}_{3}=\left[1, \frac{\sqrt{3}}{2},-\frac{1}{2}, 0\right]^{T} \\
&{ }^{0} \mathbf{r}_{B_{i}}=\mathbf{T}_{1}{ }^{1} \mathbf{r}_{B_{i}} ; \quad{ }^{0} \mathbf{r}_{C_{i}}=\mathbf{T}^{2} \mathbf{r}_{C_{i}} ; \quad{ }^{0} \mathbf{v}_{i}=\mathbf{T}^{2} \mathbf{v}_{i}, \quad i=1,2,3 .
\end{aligned}
$$




\section{Singularities of the 3-RPS-3-SPR S-PM}

It is noticed that the 3-RPS-3-SPR series-parallel manipulator can reach two kinds of singularities: a parallel singularity in which at least one of its modules is in a parallel singularity or a serial singularity ${ }^{1}$ which occurs due to the serial arrangement of the two modules. This section briefs the derivation of the forward and inverse kinematic Jacobian matrices with a hope to find out if a given configuration is singular, at least numerically. It also explains a geometrical approach to determine the singularities in which the characteristic tetrahedron ${ }^{22}$ of the S-PM under study can be expressed algebraically. The bijective mapping between the degeneracy of the tetrahedron and serial singularities can then be exploited to enlist all the serial singularities.

\subsection{Forward and inverse kinematic Jacobian matrices}

If the proximal $(P)$ and distal $(D)$ modules are considered individually, the twist i.e., angular velocity vector of a body and linear velocity vector of a point on the body, of their respective moving platform with respect to their fixed base can be expressed as a function of the actuated joint rates $^{23}$ as follows:

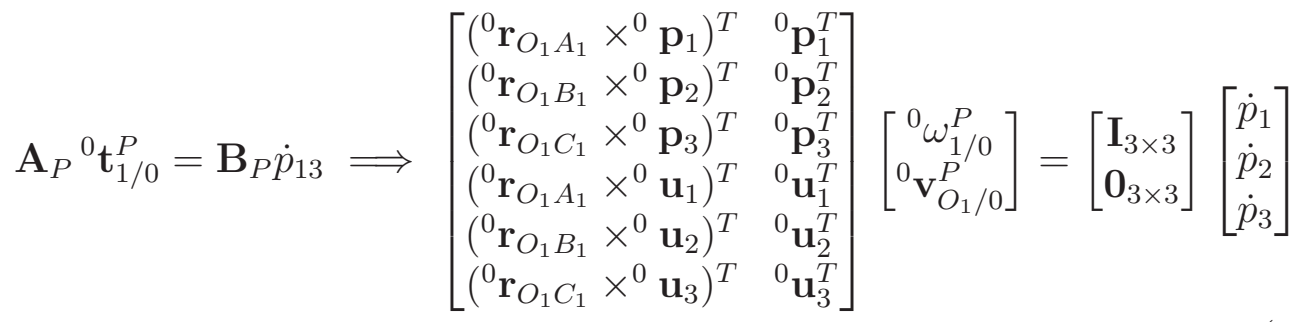

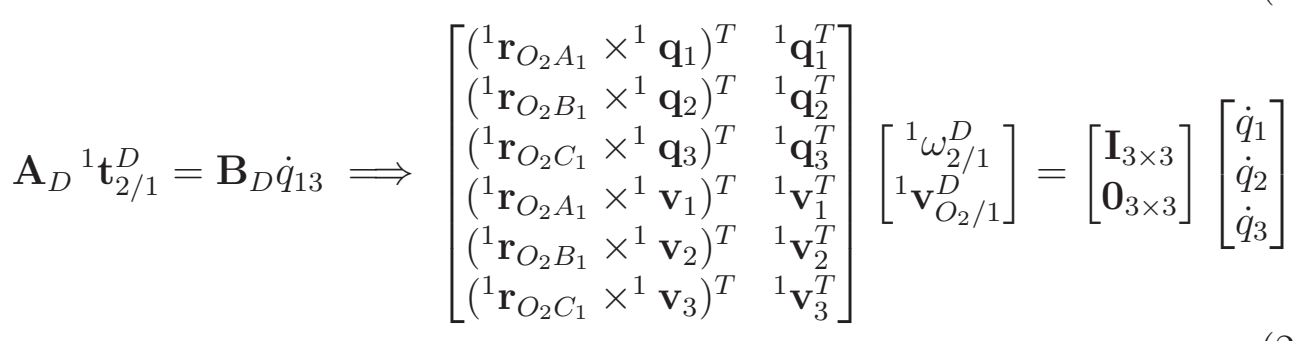

where, ${ }^{0} \mathbf{t}_{1 / 0}^{P}$ is the twist of the coupler with respect to the base expressed in $\mathcal{F}_{0}$ and ${ }^{1} \mathbf{t}_{2 / 1}^{D}$ is the twist of the end effector with respect to the coupler expressed in $\mathcal{F}_{1} . \mathbf{A}_{P}$ and $\mathbf{A}_{D}$ are called forward Jacobian matrices and they

\footnotetext{
${ }^{1}$ A serial singularity is defined here as a configuration in which the S-PM experiences a loss of degree(s) of freedom or, equivalently, a drop in the order of the twist system
} 
incorporate the actuation and constraint wrenches of the 3-RPS and 3-SPR PMs, respectively. ${ }^{23} \quad \mathbf{B}_{P}$ and $\mathbf{B}_{D}$ are called inverse Jacobian matrices and they are the result of the reciprocal product between wrenches of the mechanism and twists of the joints for the 3-RPS and 3-SPR PMs, respectively. $\dot{p}_{13}=\left[\dot{p}_{1}, \dot{p}_{2}, \dot{p}_{3}\right]^{T}$ and $\dot{q}_{13}=\left[\dot{q}_{1}, \dot{q}_{2}, \dot{q}_{3}\right]^{T}$ are the prismatic joint rates of the proximal and distal modules, respectively. ${ }^{k} \mathbf{r}_{P Q}$ denotes the vector pointing from a point $P$ to point $Q$ expressed in frame $\mathcal{F}_{k}$.

It is noteworthy that if matrix $\mathbf{A}_{P}$ (resp. $\mathbf{A}_{D}$ ) is singular, then the proximal (resp. distal) module will be in a parallel singular configuration. The entries of matrices $\mathbf{A}_{P}$ and $\mathbf{A}_{D}$ represent the Plücker coordinates of six independent lines in $\mathbb{P}^{3}$. When any two or more of these lines are dependent, the configuration corresponds to a parallel singularity. Many scientific papers deal with this singularity type of both modules. ${ }^{21,24-30}$ It is noteworthy that the 3-RPS-3-SPR S-PM is in a parallel singularity if and only if any of its modules is in a parallel singularity as proved in the following subsection.

On the other hand, due to the serial stacking of the 3-RPS and 3-SPR PMs, the S-PM can also have some serial singular configurations even if the individual modules are non-singular ${ }^{2}$. Hence, a kinematic Jacobian matrix of the S-PM is necessary to explore the serial singularities. If both $\mathbf{A}_{P}$ and $\mathbf{A}_{D}$ are nonsingular, the so called serial Jacobian matrix of the S-PM can be expressed as follows: ${ }^{17}$

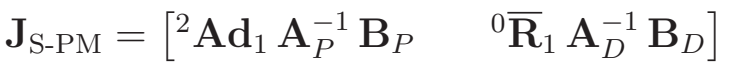

$$
\begin{aligned}
& \text { with }{ }^{2} \mathbf{A d}_{1}=\left[\begin{array}{cc}
\mathbf{I}_{3 \times 3} & \mathbf{0}_{3 \times 3} \\
-{ }^{0} \hat{\mathbf{r}}_{O_{1} O_{2}} & \mathbf{I}_{3 \times 3}
\end{array}\right],{ }^{0} \hat{\mathbf{r}}_{O_{1} O_{2}}=\left[\begin{array}{ccc}
0 & -{ }^{0} z_{O_{1} O_{2}} & { }^{0} y_{O_{1} O_{2}} \\
{ }^{0} z_{O_{1} O_{2}} & 0 & -{ }^{0} x_{O_{1} O_{2}} \\
-{ }^{0} y_{O_{1} O_{2}} & { }^{0} x_{O_{1} O_{2}} & 0
\end{array}\right] \\
& \text { and } \quad{ }^{0} \overline{\mathbf{R}}_{1}=\left[\begin{array}{cc}
{ }^{0} \mathbf{R}_{1} & \mathbf{I}_{3 \times 3} \\
\mathbf{I}_{3 \times 3} & { }^{0} \mathbf{R}_{1}
\end{array}\right]
\end{aligned}
$$

where, ${ }^{2} \mathbf{A d}_{1}$ is called the adjoint matrix. ${ }^{0} \hat{\mathbf{r}}_{O_{1} O_{2}}$ is the cross product matrix of vector ${ }^{0} \mathbf{r}_{O_{1} O_{2}}=\left[{ }^{0} x_{O_{1} O_{2}},{ }^{0} y_{O_{1} O_{2}},{ }^{0} z_{O_{1} O_{2}}\right]$, pointing from point $O_{1}$ to point $O_{2}$ expressed in frame $\mathcal{F}_{0} \cdot{ }^{0} \overline{\mathbf{R}}_{1}$ is called the augmented rotation matrix between frames $\mathcal{F}_{0}$ and $\mathcal{F}_{1}$ and it contains the rotation matrix ${ }^{0} \mathbf{R}_{1}$ from frame $\mathcal{F}_{0}$ to frame $\mathcal{F}_{1}$. $\mathbf{J}_{\mathrm{S}-\mathrm{PM}}$ fits into the kinematic model of the S-PM in the following way:

$$
{ }^{0} \mathbf{t}_{2 / 0}=\mathbf{J}_{\mathrm{S}-\mathrm{PM}}\left[\begin{array}{c}
\dot{\mathbf{p}} \\
\dot{\mathbf{q}}
\end{array}\right]
$$

where ${ }^{0} \mathbf{t}_{2 / 0}$ is the twist of the moving platform with respect to the fixed base expressed in $\mathcal{F}_{0}$ and $\dot{\mathbf{p}}=\left[\dot{p}_{1}, \dot{p}_{2}, \dot{p}_{3}\right]^{T}$ and $\dot{\mathbf{q}}=\left[\dot{q}_{1}, \dot{q}_{2}, \dot{q}_{3}\right]^{T}$ are the joint rates

\footnotetext{
${ }^{2}$ The 3 -RPS and the 3-SPR PMs do not have any serial singularities as long as the prismatic link lengths $p_{i}$ and $q_{i}, i=1,2,3$ do not vanish
} 
of the proximal and the distal modules, respectively. The rank of this matrix provides the local mobility of the S-PM. ${ }^{17}$ Moreover, when $\mathbf{J}_{S-P M}$ is singular, the series-parallel manipulator at hand is in a serial singularity. When a manipulator configuration is given, it is straightforward to calculate numerically the serial kinematic Jacobian matrix from Eq. (21) and to deduce if it is a serial singular configuration. However, it is tedious to derive a symbolic or an implicit equation that could be used to enlist all serial singularities. Therefore, a geometric approach is adopted.

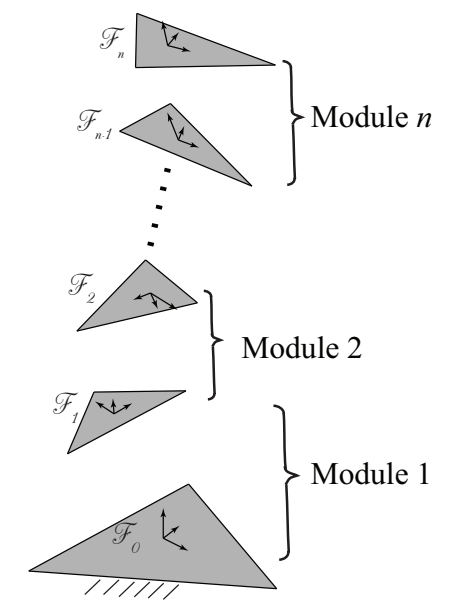

Figure 2: $n$ parallel mechanisms (named modules) arranged in series

Equations (21) and (22) can be extended to a series-parallel manipulator with $n$ number of modules in series as shown in Fig. 2. Thus, the moving platform twist with respect to the fixed base expressed in coordinate frame $\mathcal{F}_{0}$ is as follows:

$$
\begin{aligned}
& { }^{0} \mathbf{t}_{n / 0}=\sum_{i=1}^{n}{ }^{0} \overline{\mathbf{R}}_{(i-1)}{ }^{n} \mathbf{A d}_{i}{ }^{(i-1)} \mathbf{t}_{i /(i-1)}^{M_{i}}=\mathbf{J}_{6 \times 3 n}\left[\begin{array}{c}
\dot{\rho}_{M_{1}} \\
\dot{\rho}_{M_{2}} \\
\vdots \\
\dot{\rho}_{M_{n}}
\end{array}\right] \\
& \text { with } \quad{ }^{0} \overline{\mathbf{R}}_{i}=\left[\begin{array}{cc}
{ }^{0} \mathbf{R}_{i} & \mathbf{I}_{3 \times 3} \\
\mathbf{I}_{3 \times 3} & { }^{0} \mathbf{R}_{i}
\end{array}\right], \quad{ }^{n} \mathbf{A d}_{i}=\left[\begin{array}{cc}
\mathbf{I}_{3 \times 3} & \mathbf{0}_{3 \times 3} \\
-{ }^{(i-1)} \hat{\mathbf{r}}_{O_{i} O_{n}} & \mathbf{I}_{3 \times 3}
\end{array}\right] \text { and }
\end{aligned}
$$

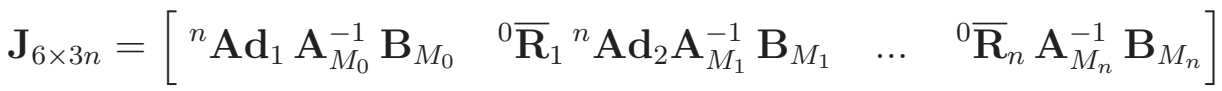


where, $\mathbf{J}_{6 \times 3 n}$ is the $6 \times 3 n$ kinematic Jacobian matrix of the $n$-module S-PM manipulator. $M_{i}$ stands for the $i$-th module, $\mathbf{A}_{M_{i}}$ and $\mathbf{B}_{M_{i}}$ are the forward and inverse Jacobian matrices of $M_{i}$, respectively. $\dot{\rho}_{M_{i}}$ is the vector of the actuated prismatic joint rates for the $i$-th module.

\subsection{Twist and wrench systems of the 3-RPS-3-SPR PM}

Each leg of the 3-RPS and 3-SPR parallel manipulators is composed of three joints, but the order of the limb twist system is equal to five and hence there exist five twists associated to each leg. Thus, the constraint wrench system of the $i$-th leg of the 3-RPS and 3-SPR parallel modules is spanned by a pure force $\mathcal{W}_{P}^{i}$ and $\mathcal{W}_{D}^{i}$ shown as the black and red vectors, respectively in Fig. 4. These forces are reciprocal to all the joint twists in each leg, in the respective modules. The three forces in each module span its wrench system $\mathcal{W}_{P}$ or $\mathcal{W}_{D}$ which is the third special three-system of screws: ${ }^{16}$

$$
\begin{aligned}
& { }^{0} \mathcal{W}_{P}=\bigoplus_{i=1}^{3}{ }^{0} \mathcal{W}_{P}^{i}=\operatorname{span}\left\{\left[\begin{array}{c}
{ }^{0} \mathbf{u}_{1} \\
{ }^{0} \mathbf{r}_{O_{2} B_{1}} \times{ }^{0} \mathbf{u}_{1}
\end{array}\right],\left[\begin{array}{c}
{ }^{0} \mathbf{u}_{2} \\
{ }^{0} \mathbf{r}_{O_{2} B_{2}} \times{ }^{0} \mathbf{u}_{2}
\end{array}\right],\left[\begin{array}{c}
{ }^{0} \mathbf{u}_{3} \\
{ }^{0} \mathbf{r}_{O_{2} B_{3}} \times{ }^{0} \mathbf{u}_{3}
\end{array}\right]\right\} \\
& { }^{0} \mathcal{W}_{D}=\bigoplus_{i=1}^{3}{ }^{0} \mathcal{W}_{D}^{i}=\operatorname{span}\left\{\left[\begin{array}{c}
{ }^{0} \mathbf{v}_{1} \\
{ }^{0} \mathbf{r}_{O_{2} B_{1}} \times{ }^{0} \mathbf{v}_{1}
\end{array}\right],\left[\begin{array}{c}
{ }^{0} \mathbf{v}_{2} \\
{ }^{0} \mathbf{r}_{O_{2} B_{2}} \times{ }^{0} \mathbf{v}_{2}
\end{array}\right],\left[\begin{array}{c}
{ }^{0} \mathbf{v}_{3} \\
{ }^{0} \mathbf{r}_{O_{2} B_{3}} \times{ }^{0} \mathbf{v}_{3}
\end{array}\right]\right\} \\
& \operatorname{dim}\left({ }^{0} \mathcal{W}_{P}\right)=\operatorname{dim}\left({ }^{0} \mathcal{W}_{D}\right)=3
\end{aligned}
$$

Alternatively, the twist system of the 3-RPS-3-SPR S-PM is the union of the twist systems of two modules. The twist systems of each module are the orthogonal vector subspaces of the respective wrench systems and are also the third special three-system of screws: ${ }^{16}$

$$
\begin{aligned}
& { }^{0} \mathcal{T}_{P}={ }^{0} \mathcal{W}_{P}^{\perp} \\
& { }^{0} \mathcal{T}_{D}={ }^{0} \mathcal{W}_{D}^{\perp} \\
& { }^{0} \mathcal{T}_{S-P M}={ }^{0} \mathcal{T}_{P} \cup{ }^{0} \mathcal{T}_{D} \\
& \operatorname{dim}\left({ }^{0} \mathcal{T}_{P}\right)=\operatorname{dim}\left({ }^{0} \mathcal{T}_{D}\right)=3
\end{aligned}
$$

The mobility of the 3-RPS-3-SPR S-PM is equal to the dimension of the overall twist system, $\operatorname{dim}\left({ }^{0} \mathcal{T}_{S-P M}\right)$. For a general configuration, when the twist systems of each module are independent i.e., $\operatorname{dim}\left({ }^{0} \mathcal{T}_{P} \cap{ }^{0} \mathcal{T}_{D}\right)=0$, the 
mobility was established to be six in [17]:

$$
\begin{aligned}
\operatorname{dim}\left({ }^{0} \mathcal{T}_{S-P M}\right)=\operatorname{dim}\left({ }^{0} \mathcal{T}_{P} \cup{ }^{0} \mathcal{T}_{D}\right) & =\operatorname{dim}\left({ }^{0} \mathcal{T}_{P}\right)+\operatorname{dim}\left({ }^{0} \mathcal{T}_{D}\right)-\operatorname{dim}\left({ }^{0} \mathcal{T}_{P} \cap{ }^{0} \mathcal{T}_{D}\right) \\
& =\operatorname{dim}\left({ }^{0} \mathcal{T}_{P}\right)+\operatorname{dim}\left({ }^{0} \mathcal{T}_{D}\right) \\
& =3+3=6
\end{aligned}
$$

As a conclusion, the following theorem is stated.

Theorem 1 A parallel singularity of an S-PM arises if and only if at least one of its modules reaches a parallel singularity.

Proof: When the actuators are blocked, the twist system of any module in a parallel singularity is of order more than zero or, equivalently, the wrench system is of order less than six. The sufficient condition is that if at least one module is in a parallel singularity, then the S-PM is in a parallel singularity. In this case, from Eq. (26), if the order of the twist system is more than zero for any module, it is reflected in the order of the twist system of the whole S-PM. The necessary condition can be proved as follows. If none of the modules is in a parallel singularity, the wrench system of each module is of order six when the actuated joints are blocked. Thus, the order of the wrench system of the full S-PM is also of order six and thus the S-PM is not in a parallel singularity.

\subsection{Enumeration of serial singularities}

A serial singularity is encountered

- when there exists a wrench common to both the modules of the S-PM or, equivalently, if the dimension of the intersection of the two wrench systems is more than zero:

$$
\operatorname{dim}\left({ }^{0} \mathcal{W}_{P} \cap{ }^{0} \mathcal{W}_{D}\right)>0
$$

- if the union of the two wrench systems is of dimension lower than six. Indeed,

$$
\begin{aligned}
\operatorname{dim}\left({ }^{0} \mathcal{W}_{P} \cup{ }^{0} \mathcal{W}_{D}\right)= & \operatorname{dim}\left({ }^{0} \mathcal{W}_{P}\right)+\operatorname{dim}\left({ }^{0} \mathcal{W}_{D}\right)-\operatorname{dim}\left({ }^{0} \mathcal{W}_{P} \cap{ }^{0} \mathcal{W}_{D}\right) \\
& =3+3-\operatorname{dim}\left({ }^{0} \mathcal{W}_{P} \cap{ }^{0} \mathcal{W}_{D}\right)
\end{aligned}
$$

From Eqs. (27) and $(28), \operatorname{dim}\left({ }^{0} \mathcal{W}_{P} \cup{ }^{0} \mathcal{W}_{D}\right)<6$ 
A straightforward sufficient condition for Eq. (27) or (28) to hold is that at least one revolute joint axis in the base is parallel to the corresponding revolute joint axis in the moving platform. In other words, ${ }^{0} \mathbf{u}_{\mathbf{i}} \|{ }^{0} \mathbf{v}_{\mathbf{i}}$ for any $i=1,2,3$. For the $i$-th leg, by equating the coordinates of vectors ${ }^{0} \mathbf{u}_{\mathbf{i}}$ and ${ }^{0} \mathbf{v}_{\mathbf{i}}$, three systems of equations are obtained from Eq. (18) in parameters $c_{1}, c_{2}, f_{1}$ and $f_{2}$. Solving the system of equations for three of the four parameters, say, $c_{1}, f_{1}$ and $c_{2}$ leads to the following algebraic expressions corresponding to the serial singular configurations:

$$
\begin{aligned}
&{ }^{0} \mathbf{u}_{\mathbf{1}} \|{ }^{0} \mathbf{v}_{\mathbf{1}} \Longrightarrow c_{1}=0, f_{1}=0 \\
&{ }^{0} \mathbf{u}_{\mathbf{2}} \|{ }^{0} \mathbf{v}_{\mathbf{2}} \Longrightarrow c_{1}=-\sqrt{3} c_{2}, f_{1}=-\sqrt{3} f_{2} \\
&{ }^{0} \mathbf{u}_{\mathbf{3}} \|{ }^{0} \mathbf{v}_{\mathbf{3}} \Longrightarrow c_{1}=\sqrt{3} c_{2}, f_{1}=\sqrt{3} f_{2} \\
&{ }^{0} \mathbf{u}_{\mathbf{i}} \|{ }^{0} \mathbf{v}_{\mathbf{i}} \quad \forall i=1,2,3 \quad \Longrightarrow \quad c_{1}=-f_{1}, c_{2}=-f_{2}
\end{aligned}
$$

When all the base revolute joint axes are parallel to their corresponding platform revolute joint axes, the fixed base and the moving platform are parallel to each other. In this case, the transformation matrix $\mathbf{T}$ is the Identity matrix resulting in $\mathbf{T}_{1}=\mathbf{T}_{2}^{-1}$. Figure 3 shows the four cases for arbitrary design parameters. In the first three cases, the constraint wrench $\tau_{0}$ prevents the moving-platform from rotating about its axis. Thus, the manipulator has only $5 \mathrm{dof}$. When the base is parallel to the moving platform, the constraint wrench system of the whole manipulator is spanned by three forces $\tau_{01}, \tau_{02}$ and $\tau_{03}$ leading to an instantaneous three dof S-PM. The degrees of freedom include a pure vertical translation and two non-pure horizontal rotations. In this case, the last condition shown in (29) can be substituted in the expression for $\mathbf{T}$ to find the coordinates of the platform circumcenter $\mathrm{O}_{2}$ to be $\left[0,0, \frac{2\left(d_{1}+g_{1}\right)}{f_{2}}\right]$, where only a $z$-translation is allowed. There can be other similar configurations in which the base and the platform are parallel with translations along all three coordinate axes. It is noteworthy that the algebraic relations governing the serial singular configurations described so far are independent of the parameters $h_{0}, h_{1}, h_{2}, g_{1}$ and $d_{1}$.

There exist other serial singular configurations in which the constraint wrenches at each spherical joint are not coincident and hence form the first special two system of screws. ${ }^{16}$ The following section describes a methodology to determine these singularities.

\subsection{Characteristic tetrahedron of serial singularities}

At each spherical joint, if the constraint forces are not coincident, they form a force pencil. A characteristic tetrahedron is defined combining the planes 


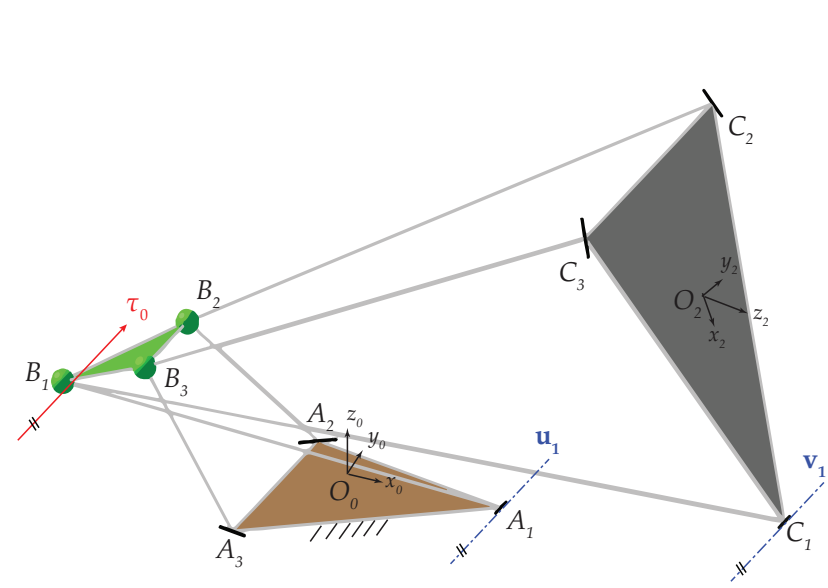

(a) ${ }^{0} \mathbf{u}_{\mathbf{1}} \|{ }^{0} \mathbf{v}_{\mathbf{1}}$

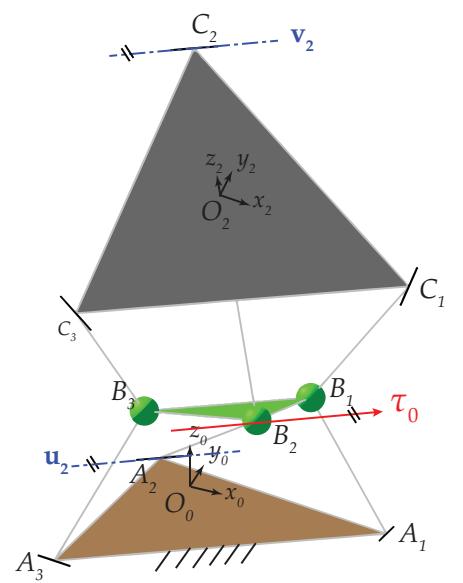

(b) ${ }^{0} \mathbf{u}_{2} \|{ }^{0} \mathbf{v}_{\mathbf{2}}$

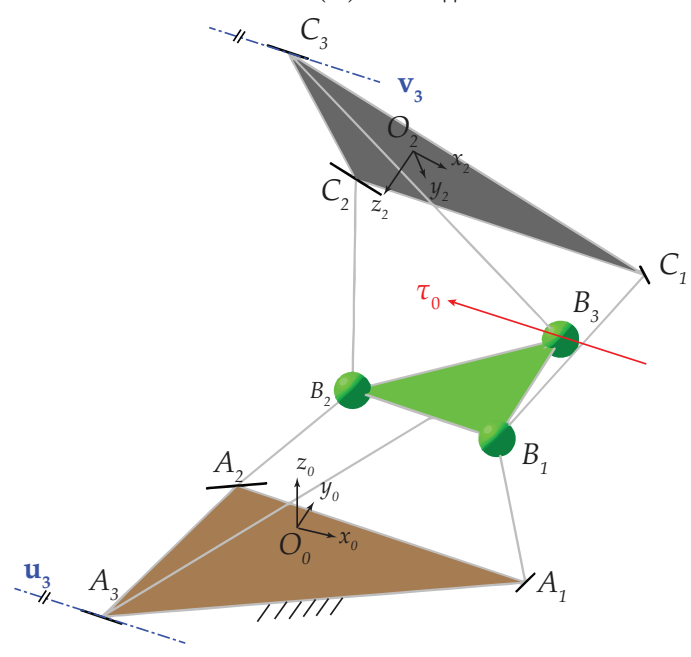

(c) ${ }^{0} \mathbf{u}_{\mathbf{3}} \|{ }^{0} \mathbf{v}_{\mathbf{3}}$

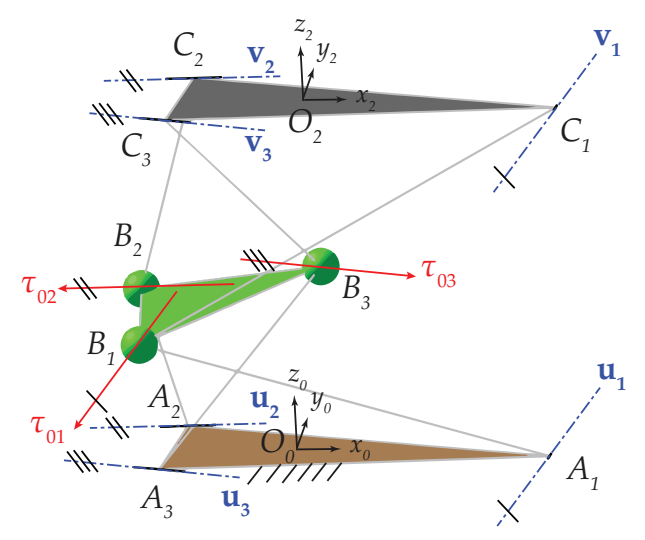

(d) ${ }^{0} \mathbf{u}_{\mathbf{i}} \|{ }^{0} \mathbf{v}_{\mathbf{i}} \forall i=1,2,3$

Figure 3: Serial singular configurations with parallel revolute joint axes 
of the three pencils along with the coupler platform plane passing through the spherical joints as shown in Fig. 4.

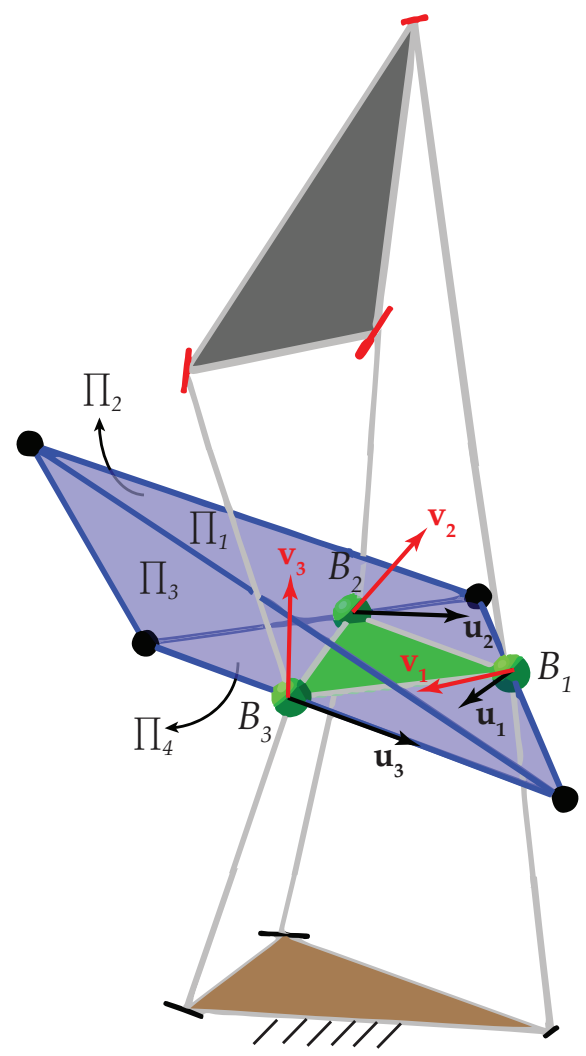

Figure 4: The characteristic tetrahedron of the 3-RPS-3-SPR S-PM

A theorem proposed by Uphoff et al.. in [22] is used to identify the remaining serial singularities.

Theorem 2 ((ref. [22])) A platform manipulator is in a wrench singularity if and only if the characteristic tetrahedron is singular

In this context, the wrench singularities correspond to the serial singularities of the S-PM. The method was initially designed to determine the parallel singularities of a parallel manipulator and the novelty of this paper lies in using the same method to enumerate serial singularities of a S-PM. The homogeneous co-ordinates of the planes (the normal vector to the plane, $\mathbf{w}_{i}$ and a point on the plane are known) representing the faces of the tetrahedron are expressed as follows:

$\Pi_{1}: \mathbf{a}=\left[w_{01}, \mathbf{w}_{1}\right]=\left[-\mathbf{r}_{B_{1}}^{T}\left(\mathbf{u}_{1} \times \mathbf{v}_{1}\right), \quad\left(\mathbf{u}_{1} \times \mathbf{v}_{1}\right)^{T}\right]$ 


$$
\begin{aligned}
& \Pi_{2}: \mathbf{b}=\left[w_{02}, \mathbf{w}_{2}\right]=\left[-\mathbf{r}_{B_{2}}^{T}\left(\mathbf{u}_{2} \times \mathbf{v}_{2}\right), \quad\left(\mathbf{u}_{2} \times \mathbf{v}_{2}\right)^{T}\right] \\
& \Pi_{3}: \mathbf{c}=\left[w_{03}, \mathbf{w}_{3}\right]=\left[-\mathbf{r}_{B_{3}}^{T}\left(\mathbf{u}_{3} \times \mathbf{v}_{3}\right), \quad\left(\mathbf{u}_{3} \times \mathbf{v}_{3}\right)^{T}\right] \\
& \Pi_{4}: \mathbf{d}=\left[w_{04}, \mathbf{w}_{4}\right] \\
& =\left[-\mathbf{r}_{B_{1}}^{T}\left(\left(\mathbf{r}_{B_{1}}-\mathbf{r}_{B_{2}}\right) \times\left(\mathbf{r}_{B_{1}}-\mathbf{r}_{B_{3}}\right)\right), \quad\left(\left(\mathbf{r}_{B_{1}}-\mathbf{r}_{B_{2}}\right) \times\left(\mathbf{r}_{B_{1}}-\mathbf{r}_{B_{3}}()^{3}\right)^{\top}\right]\right]
\end{aligned}
$$

All the vectors are expressed in frame $\mathcal{F}_{0}$. Hence, a serial singularity occurs when the determinant of the matrix of plane coordinates $[\mathbf{a} \mathbf{b} \mathbf{c} \mathbf{d}]$ vanishes. A similar approach using Grassman-Cayley algebra was used to find singularities of a six-dof manipulator, 3-PPPS in [31]. From Eqs. (18) and (30),

$$
\begin{aligned}
& |\mathbf{a} \mathbf{b} \mathbf{c} \mathbf{d}|=-\frac{27\left(c_{1} f_{1}+c_{2} f_{2}-1\right) h_{1}{ }^{3}}{2\left(c_{1}{ }^{2}+c_{2}{ }^{2}+1\right)^{3}\left(f_{1}{ }^{2}+f_{2}{ }^{2}+1\right)^{3}}\left(4 c_{1}^{4} f_{1} f_{2}{ }^{2}-8 c_{1}{ }^{3} c_{2} f_{1}{ }^{2} f_{2}-8 c_{1}{ }^{3} c_{2} f_{2}{ }^{3}\right. \\
& +4 c_{1}^{3} f_{1}^{2} f_{2}^{2}-4 c_{1}^{3} f_{2}^{4}+4 c_{1}^{2} c_{2}^{2} f_{1}^{3}+12 c_{1}^{2} c_{2}^{2} f_{1} f_{2}{ }^{2}-8 c_{1}^{2} c_{2} f_{1}^{3} f_{2} \\
& +4 c_{1} c_{2}^{2} f_{1}^{4}+12 c_{1} c_{2}^{2} f_{1}^{2} f_{2}^{2}-4 c_{2}^{4} f_{1}^{3}-8 c_{2}^{3} f_{1}^{3} f_{2}+c_{1}^{4} f_{1}-8 c_{1}^{3} c_{2} f_{2} \\
& +3 c_{1}^{3}{f_{1}}^{2}-3 c_{1}^{3}{f_{2}}^{2}+6 c_{1}^{2} c_{2}^{2} f_{1}-6 c_{1}^{2} c_{2} f_{1} f_{2}+3 c_{1}^{2} f_{1}^{3}+3 c_{1}^{2} f_{1} f_{2}^{2} \\
& +3 c_{1} c_{2}^{2} f_{1}^{2}-3 c_{1} c_{2}^{2} f_{2}^{2}-6 c_{1} c_{2} f_{1}^{2} f_{2}-6 c_{1} c_{2} f_{2}^{3}+c_{1} f_{1}^{4}+6 c_{1} f_{1}^{2} f_{2}^{2} \\
& -3 c_{1} f_{2}{ }^{4}-3 c_{2}^{4} f_{1}-6 c_{2}^{3} f_{1} f_{2}-3 c_{2}^{2} f_{1}^{3}-3 c_{2}^{2} f_{1} f_{2}^{2}-8 c_{2} f_{1}^{3} f_{2}+c_{1}^{3} \\
& +3 c_{1}^{2} f_{1}-3 c_{1} c_{2}^{2}-6 c_{1} c_{2} f_{2}+3 c_{1} f_{1}^{2}-3 c_{1} f_{2}^{2}-3 c_{2}^{2} f_{1}-6 c_{2} f_{1} f_{2} \\
& \left.+f_{1}{ }^{3}-3 f_{1}{f_{2}}^{2}\right)
\end{aligned}
$$

Thus, the points on the surface $|\mathbf{a} \mathbf{b} \mathbf{c} \mathbf{d}|=0$ correspond to serial singular configurations for the 3-RPS-3-SPR S-PM. From Eq. (31), the manipulator is in a serial singular configuration when either $c_{1} f_{1}+c_{2} f_{2}-1=0$ or the second factor, a 7 degree polynomial, $p^{7}\left(c_{1}, f_{1}, c_{2}, f_{2}\right)=0^{3}$. In order to enumerate different serial singularities, the conditions for rank deficiency of the matrix [a b c d] listed in Table 1 of [22] are studied.

Case 1: 4 faces meet in a point Two subcases must be considered depending on whether the point of intersection is real or lies at infinity. In both cases, the variety spanned by the 6 constraint wrench lines is a general linear complex ${ }^{32}$ and the 3-RPS-3-SPR S-PM instantaneously behaves as a 5 dof mechanism.

a. A real point: Considering the second factor of Eq. (31) $p^{7}\left(c_{1}, c_{2}, f_{1}, f_{2}\right)$, substituting arbitrary values for any three of the four parameters and finding

\footnotetext{
${ }^{3} \mathrm{~A} 3 \mathrm{D}$ animation of the singular surface by varying $c_{1}, c_{2}, f_{1}$ and $f_{2}$ from -3 to 3 is uploaded in https://www.dropbox.com/s/dzif65bhx59nxd6/sing1.mp4?dl=0 for the first factor of Eq. (31) and in https://www.dropbox.com/s/koezrl6xom3pmmr/singp7.mp4?dl=0 for the second factor of Eq. (31).
} 
the fourth one shows that the faces of the characteristic tetrahedron intersect in a point. One such configuration is shown in Fig. 5a. Point $P$ is the intersection point of the four planes $\Pi_{j}, j=1,2,3,4$.

b. A point at infinity: This happens when the intersection lines of the planes are parallel. In other words, it is sufficient to check if the ideal point (nothing but the point at infinity of a line) of one of these lines lies in the other three planes. Let the ideal point of line of intersection, $L_{12}$, of planes $\Pi_{1}$ and $\Pi_{2}$ be $P_{12}^{\infty}$. It is sufficient to check if this point lies on the line of intersection, $L_{34}$, of planes $\Pi_{3}$ and $\Pi_{4}$. However, this approach is computationally expensive and yields no results. Therefore, it is first checked whether point $P_{12}^{\infty}$ lies on the line of intersection, $L_{13}$, of planes $\Pi_{1}$ and $\Pi_{3}$ as follows:

$$
\begin{aligned}
\mathbf{r}_{P_{12}^{\infty}} & :\left(0, \mathbf{l}_{12}\right)=\left(0, \mathbf{w}_{1} \times \mathbf{w}_{2}\right) \\
L_{13} & :\left(\mathbf{l}_{13}, \overline{\mathbf{l}}_{13}\right)=\left(\mathbf{w}_{1} \times \mathbf{w}_{3}, w_{01} \mathbf{w}_{3}-w_{03} \mathbf{w}_{1}\right) \\
\mathbf{r}_{P_{12}^{\infty}}^{\infty} \wedge L_{12}=0 & : \mathbf{w}_{3} \cdot \mathbf{l}_{12}=0, \quad-w_{03} \mathbf{l}_{12}+\mathbf{w}_{3} \times \overline{\mathbf{l}}_{12}=0
\end{aligned}
$$

Solving Eq. (34) for $c_{1}, f_{1}, c_{2}$ and $f_{2}$ yields the relationship $c_{1} f_{1}+c_{2} f_{2}-1=0$ or $c_{1} f_{2}-c_{2} f_{1}=0$. The former relationship corresponds to the intersection of the planes in a real line. It will be discussed in the following paragraph. The latter corresponds to the configuration where the planes $\Pi_{1}, \Pi_{2}$ and $\Pi_{3}$ share the same ideal point. It can also mean that they have a common line of intersection at infinity, which will be dealt with in the next paragraph. In other words, their lines of intersection are parallel. Formulating another equation such that the point $P_{12}^{\infty}$ lies in the plane $\Pi_{4}$ and solving the two equations results in the following relationships:

$$
\left.\begin{array}{l}
c_{1} f_{2}-c_{2} f_{1}=0 \\
\mathbf{r}_{P_{12}^{\infty}} \cdot\left(\left[w_{04}, \mathbf{w}_{4}\right]\right)=0
\end{array}\right\} \Longrightarrow \begin{aligned}
& f_{1}=-\frac{c_{1}}{c_{1}^{2}+c_{2}^{2}}, f_{2}=-\frac{c_{2}}{c_{1}^{2}+c_{2}^{2}} \\
& c_{1}=-\frac{f_{1}}{f_{1}^{2}+f_{2}^{2}}, c_{2}=-\frac{f_{2}}{f_{1}^{2}+f_{2}^{2}}
\end{aligned}
$$

If the parameters $c_{1}, c_{2}, f_{1}$ and $f_{2}$ satisfy the foregoing conditions, the S$\mathrm{PM}$ is in a serial singularity with the planes of its characteristic tetrahedron intersecting in a point at infinity. One such configuration is depicted in Fig. 5b.

Case 2: 3 sides meet in a line : The first factor of Eq. (31), $c_{1} f_{1}+c_{2} f_{2}-$ $1=0$ corresponds to the serial singularity in which the variety spanned by the 6 constraint wrench lines is a special linear complex ${ }^{32}$ and the mechanism 


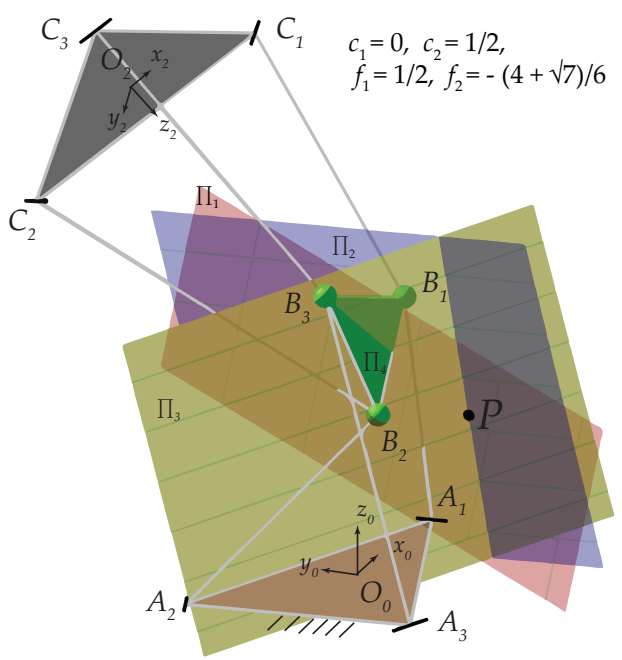

(a) A real point $P$

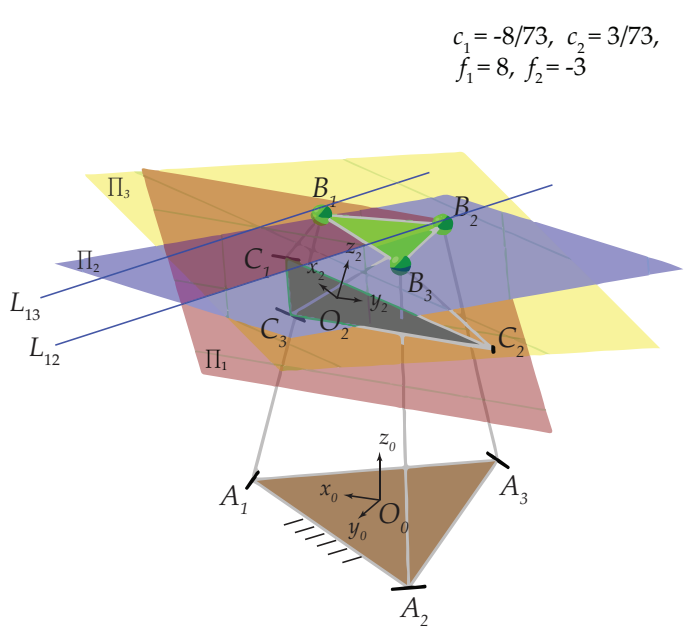

(b) A point at infinity

Figure 5: Serial singularity when all faces of the characteristic tetrahedron meet in a point

has 5 dof. To prove that $c_{1} f_{1}+c_{2} f_{2}=1$ corresponds to the singularity when 3 sides of the characteristic tetrahedron meet in a line, two sub cases are considered when the line of intersection of the three sides is

a. A real line: The condition $c_{1} f_{1}+c_{2} f_{2}-1=0$ is derived using line geometry. For a line intersection of the three planes, it is sufficient to prove the incidence of the intersection line of first two sides with the third one. The Plücker coordinates of the line of intersection, $L_{12}$ of planes $\Pi_{1}$ and $\Pi_{2}$ are calculated. The line $L_{12}$ and the plane $\Pi_{3}$ are incident if and only if the following conditions are satisfied. ${ }^{33}$

$$
\begin{array}{rll}
L_{12} & : & \left(\mathbf{l}_{12}, \overline{\mathbf{l}}_{12}\right)=\left(\mathbf{w}_{1} \times \mathbf{w}_{2}, w_{01} \mathbf{w}_{2}-w_{02} \mathbf{w}_{1}\right) \\
\Pi_{3} \wedge L_{12}=0 & : & \mathbf{w}_{3} \cdot \mathbf{l}_{12}=0, \quad-w_{03} \mathbf{l}_{12}+\mathbf{w}_{3} \times \overline{\mathbf{l}}_{12}=0
\end{array}
$$

The four equations in Eq. (37) are solved for parameters $c_{1}, c_{2}, f_{1}$ and $f_{2}$ to obtain the solution $f_{2}=-\frac{c_{1} f_{1}-1}{c_{2}}$ with arbitrary values for $c_{1}, c_{2}$ and $f_{1}$. It means that if the choice of these parameters are bound by the relation $c_{1} f_{1}+c_{2} f_{2}-1=0$, the three sides intersect in a line and is consistent with the first factor of Eq. (31). Figure 6a shows one of the serial singular configurations in which the three sides meet in a line $L$. It implies that six constraint forces intersect the line $L$ and hence belong to a singular linear line complex.

b. A line at infinity: In this case, the side planes are all parallel to each 


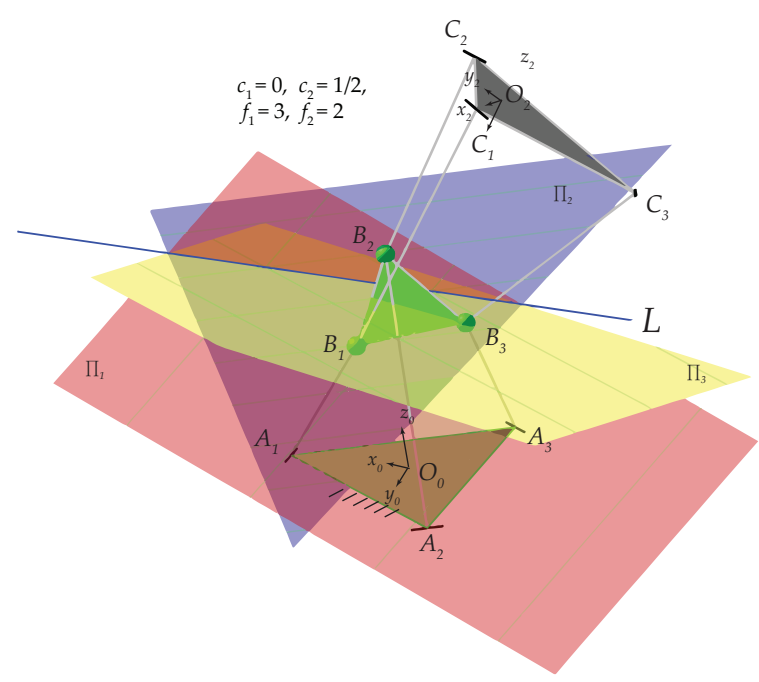

(a) A real line $L$

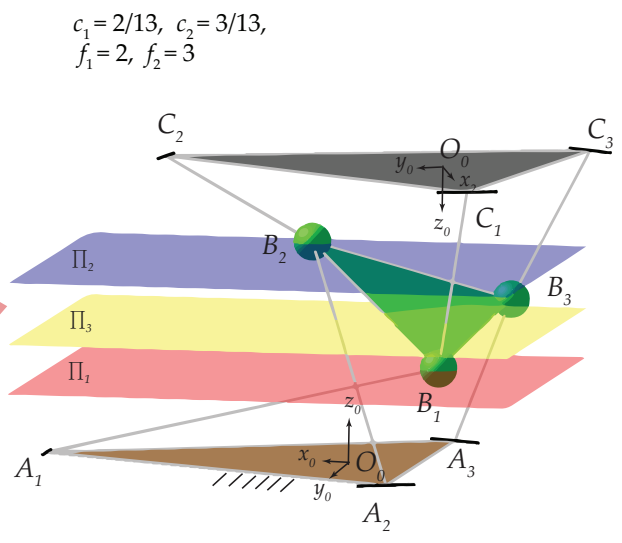

(b) A line at infinity

Figure 6: Serial singular configurations when three sides of the characteristic tetrahedron meet in a line

other which is possible only when the fixed base and the moving platform planes are parallel to each other. Since the 3-RPS-3-SPR PM has 6 dof, the only possibilities for the platform and the base to remain parallel is when the moving platform has pure translational motions or has a rotation about the $z_{0}$-axis along with translational motions. The former case is studied in Subsec. 4.3 where, corresponding revolute joint axes are parallel to each other leading to a 3 dof freedom mechanism. The latter case is investigated by considering the transformation matrix $\mathbf{T}$, and forcing the rotation matrix to be of pure rotation about $z_{0}$-axis. This is done by equating $\mathbf{T}(2,4)$ and $\mathbf{T}(3,4)$ to zero and solving for two of the four parameters $c_{1}, c_{2}, f_{1}$ and $f_{2}$ :

$$
\left.\begin{array}{l}
\mathbf{T}(2,4)=-\mathbf{T}(4,2)=0 \\
\mathbf{T}(3,4)=-\mathbf{T}(4,3)=0
\end{array}\right\} \Longrightarrow \begin{gathered}
f_{1}=\frac{c_{1}}{c_{1}^{2}+c_{2}^{2}}, f_{2}=\frac{c_{2}}{c_{1}^{2}+c_{2}^{2}} \\
\quad \begin{array}{c}
O R \\
c_{1}=\frac{f_{1}}{f_{1}^{2}+f_{2}^{2}}, c_{2}=\frac{f_{2}}{f_{1}^{2}+f_{2}^{2}}
\end{array}
\end{gathered}
$$

The relations in Eq. (38) satisfy the equation $c_{1} f_{1}+c_{2} f_{2}-1=0$. Hence, this equation is a necessary and a sufficient condition for three sides to have a common line of intersection and it instantaneously reduces the degree of freedom of the S-PM at hand by 1. Furthermore, by substituting 


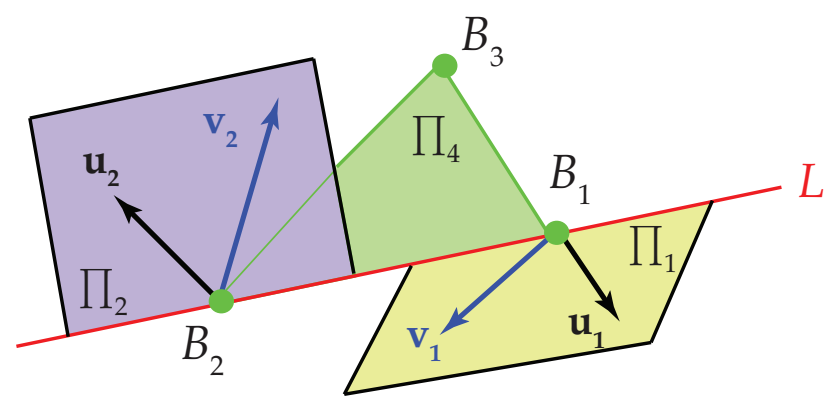

Figure 7: Can two sides and base of the characteristic tetrahedron of the 3-RPS-3-SPR S-PM meet in a line?

Eq. (38) in $\mathbf{T}$, the magnitude of rotation about the $z_{0}$-axis is given by $\sigma=\tan ^{-1}\left(\frac{2 c_{1} c_{2}}{c_{2}^{2}-c_{1}^{2}}\right)=\tan ^{-1}\left(\frac{2 f_{1} f_{2}}{f_{2}^{2}-f_{1}^{2}}\right)$. A serial singular configuration in which $\sigma=67^{\circ}$ is shown in Fig. 6b. Note that in theory the platform can have an upright position or an upside down position and yet stay parallel to the base.

Case 3: 2 sides and base meet in a line Considering a side plane $\Pi_{i}, i=1,2,3$ and the base plane $\Pi_{4}$, their line of intersection must pass through $B_{i}$. To prove if $\Pi_{i}, \Pi_{j}$ and $\Pi_{4}, i, j=1,2,3$ have a common line of intersection, it is sufficient to prove that the points $B_{i}$ and $B_{j}$ simultaneously lie on the planes $\Pi_{j}$ and $\Pi_{i}$, respectively. If there exists a line common to all the three planes, it should be along $B_{i} B_{j}$ as shown in Fig. 7. For instance, if planes $\Pi_{1}, \Pi_{2}$ and $\Pi_{4}$ are considered, simultaneous incidence of point $B_{1}$ on $\Pi_{2}$ and that of point $B_{2}$ on $\Pi_{1}$ must be satisfied and is expressed by the following equations:

$$
\begin{aligned}
& \mathbf{r}_{B_{1}} \cdot\left(\left[w_{02}, \mathbf{w}_{2}\right]\right)=0 \Longrightarrow \\
& -2 \sqrt{3} c_{1} c_{2} f_{1}-2 \sqrt{3} c_{1} f_{1} f_{2}+3 c_{1}{ }^{2} f_{1}+3 f_{1}{ }^{2} c_{1}-3 f_{2}{ }^{2} c_{1}-3 c_{2}{ }^{2} f_{1}-3 c_{1}-3 f_{1}=0 \\
& \mathbf{r}_{B_{2}} \cdot\left(\left[w_{01}, \mathbf{w}_{1}\right]\right)=0 \Longrightarrow \\
& -4 \sqrt{3} c_{1} c_{2} f_{2}-4 \sqrt{3} c_{2} f_{1} f_{2}+4 c_{2} c_{1} f_{1}+4 f_{2} c_{1} f_{1}+c_{1} \sqrt{3}+\sqrt{3} f_{1}-3 c_{2}-3 f_{2}=0
\end{aligned}
$$

Finding the Groebner basis of the polynomials in Eqs. (39) and (40) with a graded reverse lexicographic ordering (tdeg in Maple) of the parameters $c_{1}<_{\text {grlex }} c_{2}<_{\text {grlex }} f_{1}<_{\text {grlex }} f_{2}$, results in a basis of four polynomials. The four equations can then be solved for $c_{1}, c_{2}, f_{1}$ and $f_{2}$. Two solutions are 
obtained, the first one turns out to be a complex solution and the second one is exactly the last case of (29). The second solution should also be rejected since it is assumed for this analysis that none of the faces of the characteristic tetrahedron degenerates into a line. Also for other combinations of sides and their intersections with the base plane, the following equations are solved for the parameters:

$$
\begin{aligned}
& \mathbf{r}_{B_{2}} \cdot\left(\left[w_{03}, \mathbf{w}_{3}\right]\right)=0 \Longrightarrow \\
& 3 c_{1}^{2} f_{2}-2 c_{1} c_{2} f_{1}-2 c_{1} f_{1} f_{2}-3 c_{2}^{2} f_{2}+3 c_{2} f_{1}^{2}-3 c_{2} f_{2}^{2}+3 c_{2}+3 f_{2}=0 \\
& \mathbf{r}_{B_{3}} \cdot\left(\left[w_{02}, \mathbf{w}_{2}\right]\right)=0 \Longrightarrow \\
& c_{1}^{2} f_{1}-2 c_{1} c_{2} f_{2}+c_{1} f_{1}^{2}-c_{1} f_{2}{ }^{2}-c_{2}{ }^{2} f_{1}-2 c_{2} f_{1} f_{2}+c_{1}+f_{1}=0 \\
& \mathbf{r}_{B_{1}} \cdot\left(\left[w_{03}, \mathbf{w}_{3}\right]\right)=0 \Longrightarrow \\
& -4 \sqrt{3} c_{1} c_{2} f_{2}-4 \sqrt{3} c_{2} f_{1} f_{2}-4 c_{1} c_{2} f_{1}-4 c_{1} f_{1} f_{2}+c_{1} \sqrt{3}+\sqrt{3} f_{1}+3 c_{2}+3 f_{2}=0 \\
& \mathbf{r}_{B_{3}} \cdot\left(\left[w_{01}, \mathbf{w}_{1}\right]\right)=0 \Longrightarrow \\
& 2 \sqrt{3} c_{1} c_{2} f_{1}+2 \sqrt{3} c_{1} f_{1} f_{2}+3 c_{1}^{2} f_{1}+3 c_{1} f_{1}{ }^{2}-3 c_{1} f_{2}{ }^{2}-3 c_{2}{ }^{2} f_{1}-3 c_{1}-3 f_{1}=0
\end{aligned}
$$

In each case, the solutions obtained are either complex or correspond to the last case of (29), showing that the S-PM at hand cannot have a configuration in which any two sides and the base of its characteristic tetrahedron meet in a line.

Another approach to solve this case is by finding the condition for incidence of an intersection line between two sides and the base: ${ }^{33}$

$$
\begin{array}{rll}
L_{i j} & : & \left(\mathbf{l}_{i j}, \overline{\mathbf{l}}_{i j}\right)=\left(\mathbf{w}_{i} \times \mathbf{w}_{j}, w_{0 i} \mathbf{w}_{j}-w_{0 j} \mathbf{w}_{i}\right) \\
\Pi_{4} \wedge L_{i j}=0 & : & \mathbf{w}_{4} \cdot \mathbf{l}_{i j}=0, \quad-w_{03} \mathbf{l}_{i j}+\mathbf{w}_{4} \times \overline{\mathbf{l}}_{i j}=0, \quad i=1,2,3
\end{array}
$$

Equation (46) does not yield any real or non-trivial solutions. As a result, it is proved by contradiction that the 3-RPS-3-SPR S-PM cannot have a serial singular configuration in which any two sides and the base of the characteristic tetrahedron meet in a line.

Cases 4 and above The remaining cases in Table 1 of [22] include two sides meet in a plane, one side and base meet in a plane, two sides and base meet in a plane, two faces meet in a plane. Since the S-PM cannot attain a 
Table 1: Enumeration of serial singularities for the 3-RPS-3-SPR S-PM

\begin{tabular}{|c|c|c|c|}
\hline Geometrical condition & Algebraic expression & Inst.dof & Config. ex. \\
\hline i. ${ }^{0} \mathbf{u}_{\mathbf{1}} \|{ }^{0} \mathbf{v}_{\mathbf{1}}$ & $c_{1}=0, f_{1}=0$ & 5 & Fig. 3a \\
\hline ii. ${ }^{0} \mathbf{u}_{\mathbf{2}} \|{ }^{0} \mathbf{v}_{\mathbf{2}}$ & $c_{1}=-\sqrt{3} c_{2}, f_{1}=-\sqrt{3} f_{2}$ & 5 & Fig. $3 b$ \\
\hline iii. ${ }^{0} \mathbf{u}_{\mathbf{3}} \|{ }^{0} \mathbf{v}_{\mathbf{3}}$ & $c_{1}=\sqrt{3} c_{2}, f_{1}=\sqrt{3} f_{2}$ & 5 & Fig. 3c \\
\hline $\begin{array}{cc} & \text { parallel base and platform } \\
\text { iv. } & \text { (platform pure translation) } \\
& { }^{0} \mathbf{u}_{\mathbf{i}} \|{ }^{0} \mathbf{v}_{\mathbf{i}} \quad \forall i=1,2,3\end{array}$ & $c_{1}=-f_{1}, c_{2}=-f_{2}$ & 3 & Fig. 3d \\
\hline
\end{tabular}

\section{Degeneracy of the characteristic tetrahedron ${ }^{22}$}

vi. 4 faces meet in a point

(general linear complex ${ }^{32}$ )

a. a real point

b. a point at infinity

$$
p^{7}\left(c_{1}, c_{2}, f_{1}, f_{2}\right)=0 \text { (Eq. (31)) }
$$

Fig. $5 \mathrm{a}$

$c_{1}=-\frac{f_{1}}{f_{1}^{2}+f_{2}^{2}}, c_{2}=-\frac{f_{2}}{f_{1}^{2}+f_{2}^{2}}$

Fig. 5b

3 sides meet in a line

vi. $\quad \begin{aligned} & 3 \text { sides meet in a line } \\ & \text { (special linear complex) }\end{aligned}$

a. a real line

$$
c_{1} f_{1}+c_{2} f_{2}-1=0
$$

Fig. 6a

b. a line at infinity

parallel base and platform

(rotation about $z_{0}$-axis)

$$
c_{1}=\frac{f_{1}}{f_{1}^{2}+f_{2}^{2}}, c_{2}=\frac{f_{2}}{f_{1}^{2}+f_{2}^{2}}
$$

Fig. 6b

configuration of Case 3, it is certain that it cannot reach any configuration corresponding to the remaining cases. For example, if two sides could meet in a plane, this case should have appeared as a solution to Eqs. (39) and (40) and in which case, there definitely would have existed a line of intersection between the meeting plane and the base.

To this end, all possible serial singularities are listed in Tab. 1

It is recalled here that the singularity analysis performed in this section is by considering both modules in the operation mode corresponding to $c_{3}=f_{3}=0$. In fact, there are three other possibilities, $c_{0}=f_{0}=0$, $c_{0}=f_{3}=0$ and $c_{3}=f_{0}=0$. For these cases, the algebraic expressions for serial singularities can be obtained by the following replacements to the 
ones listed in Tab. 1. These replacements hold true only for the orientation parameters. Favorably, the serial singular configurations for $f_{3}=c_{3}=0$ expressed in Tab. 1 are only functions of orientation parameters $c_{i}, f_{i}, i=1,2$.

$$
\begin{array}{lll}
\text { Case } & a . & c_{3}=f_{3}=0: \text { Listed in Tab. } 1 \\
\text { Case } & \text { b. } & c_{0}=f_{0}=0: f_{2} \rightarrow-f_{1}, f_{1} \rightarrow f_{2}, c_{1} \rightarrow-c_{2}, c_{2} \rightarrow c_{1} \\
\text { Case } & \text { c. } & c_{0}=f_{3}=0: c_{1} \rightarrow-c_{2}, c_{2} \rightarrow c_{1} \\
\text { Case } & \text { d. } & c_{0}=f_{3}=0: f_{2} \rightarrow-f_{1}, f_{1} \rightarrow f_{2}
\end{array}
$$

Proof: For the 3 -RPS $\left(f_{i}, i=0,1,2,3\right)$ and the 3 -SPR $\left(c_{i}, i=0,1,2,3\right)$ parallel manipulator modules, the orientation Study parameters can be expressed in terms of the Tilt and Torsion angles, ${ }^{34}$ azimuth $(\phi)$, tilt $(\theta)$ and torsion $(\sigma)$ as follows:

$$
\begin{aligned}
f_{0} & =\cos \left(\frac{\theta_{1}}{2}\right) \cos \left(\frac{\sigma_{1}}{2}\right) & c_{0} & =\cos \left(\frac{\theta_{2}}{2}\right) \cos \left(\frac{\sigma_{2}}{2}\right) \\
f_{1} & =\sin \left(\frac{\theta_{1}}{2}\right) \cos \left(\phi_{1}-\frac{\sigma_{1}}{2}\right) & c_{1} & =-\sin \left(\frac{\theta_{2}}{2}\right) \cos \left(\phi_{2}-\frac{\sigma_{2}}{2}\right) \\
f_{2} & =\sin \left(\frac{\theta_{1}}{2}\right) \sin \left(\phi_{1}-\frac{\sigma_{1}}{2}\right) & c_{2} & =-\sin \left(\frac{\theta_{2}}{2}\right) \sin \left(\phi_{2}-\frac{\sigma_{2}}{2}\right) \\
f_{3} & =\cos \left(\frac{\theta_{1}}{2}\right) \sin \left(\frac{\sigma_{1}}{2}\right) & c_{3} & =-\cos \left(\frac{\theta_{2}}{2}\right) \sin \left(\frac{\sigma_{2}}{2}\right)
\end{aligned}
$$

The operation mode $c_{0}=0$ or $f_{0}=0$ renders the torsion angle $\sigma_{1}=0$ or $\sigma_{2}=0$ and if $f_{3}=0$ or $c_{3}=0, \sigma_{1}=180^{\circ}$ or $\sigma_{2}=180^{\circ}$, respectively. Furthermore, if, for instance in the operation mode corresponding to $f_{0}=0$, the 3 -RPS PM can never have $f_{3}=0$, thus the parameters $f_{i}, i=0,1,2,3$ can be normalized by forcing $f_{3}=1$. Consequently, the operation modes $(\mathrm{OM})$ as functions of tilt and azimuth angles can be represented for each module as follows:

\section{3-RPS OM-1}

$$
\begin{aligned}
& f_{0}=1 \\
& f_{1}=\tan \left(\frac{\theta_{1}}{2}\right) \cos \left(\phi_{1}\right) \\
& f_{2}=\tan \left(\frac{\theta_{1}}{2}\right) \sin \left(\phi_{1}\right) \\
& f_{3}=0
\end{aligned}
$$

\section{3-SPR OM-1}

$$
\begin{aligned}
c_{0} & =1 \\
c_{1} & =-\tan \left(\frac{\theta_{2}}{2}\right) \cos \left(\phi_{2}\right) \\
c_{2} & =-\tan \left(\frac{\theta_{2}}{2}\right) \sin \left(\phi_{2}\right) \\
c_{3} & =0
\end{aligned}
$$




\section{3-RPS OM-2}

$$
\begin{aligned}
& f_{0}=0 \\
& f_{1}=\tan \left(\frac{\theta_{1}}{2}\right) \sin \left(\phi_{1}\right) \\
& f_{2}=-\tan \left(\frac{\theta_{1}}{2}\right) \cos \left(\phi_{1}\right) \\
& f_{3}=1
\end{aligned}
$$

\section{3-SPR OM-2}

$$
\begin{aligned}
& c_{0}=0 \\
& c_{1}=-\tan \left(\frac{\theta_{2}}{2}\right) \sin \left(\phi_{2}\right) \\
& c_{2}=\tan \left(\frac{\theta_{2}}{2}\right) \cos \left(\phi_{2}\right) \\
& c_{3}=1
\end{aligned}
$$

Thus, it is obvious that for the 3-RPS PM, algebraic expressions in OM-2 can be obtained from those in OM-1 by replacing $f_{2} \rightarrow-f_{1}$ and $f_{1} \rightarrow f_{2}$. For the 3-SPR PM, these replacements will be $c_{2} \rightarrow c_{1}$ and $c_{1} \rightarrow-c_{2}$. Accordingly, all the serial singular configurations of the 3-RPS-3-SPR PM can be enumerated starting from Case a (where both modules are in OM-1) of Eq. (47).

\section{$5 \quad$ Direct Kinematics Model(DKM)}

The six prismatic joints of the 3-RPS-3-SPR S-PM are assumed to be actuated. Direct kinematics gives the pose, i.e., the position and orientation, of the moving platform for given prismatic joint lengths. For the 3-RPS and the 3-SPR PMs, the prismatic joint lengths are named as $p_{1}, p_{2}, p_{3}$ and $q_{1}, q_{2}, q_{3}$, respectively. If Study parameters $x_{0}, x_{1}, x_{2}, x_{3}, y_{0}, y_{1}, y_{2}$ and $y_{3}$ represent the pose of the moving platform relative to the base, the direct kinematics problem aims to find $x_{i}$ and $y_{i}, i=0,1,2,3$ given $p_{j}$ and $q_{j}, j=1,2,3$.

As mentioned in Sec. 3, the transformation matrix between the fixed base and the moving platform, $\mathbf{T}$ is determined to be a function of $f_{1}, f_{2}, g_{1}, c_{1}, c_{2}$ and $d_{1}$ for the case $c_{3}=f_{3}=0$. Therefore, the sphere constraint equations $^{21}$ for each module after factoring out the non-zero terms are expressed as follows:

$$
\begin{aligned}
& \left\|{ }^{0} \mathbf{r}_{B_{i}}-{ }^{0} \mathbf{r}_{A_{i}}\right\|^{2}=p_{i}^{2} \quad i=1,2,3 \Longrightarrow \\
& S_{1}:=\left(-p_{1}^{2}+h_{0}^{2}-4 h_{0} h_{1}+4 h_{1}^{2}\right) f_{1}^{4} f_{2}^{2}+4 f_{1}^{4} g_{1}^{2}+4 h_{1} f_{1}^{4} g_{1}+h_{1}^{2} f_{1}^{4} \\
& +\left(-2 p_{1}{ }^{2}+2 h_{0}{ }^{2}-8 h_{1}{ }^{2}\right) f_{1}{ }^{2} f_{2}{ }^{4}+8 f_{1}{ }^{2}{f_{2}}^{2} g_{1}{ }^{2}-8 h_{1} f_{1}{ }^{2} f_{2}{ }^{2} g_{1}+\left(-2 p_{1}{ }^{2}\right. \\
& \left.+2 h_{0}^{2}-6 h_{0} h_{1}-2 h_{1}^{2}\right) f_{1}^{2}{f_{2}}^{2}+8 f_{1}^{2} g_{1}^{2}+4 h_{1} f_{1}^{2} g_{1}+\left(-p_{1}^{2}+h_{0}^{2}\right. \\
& \left.+4 h_{0} h_{1}+4 h_{1}{ }^{2}\right) f_{2}{ }^{6}+4 f_{2}{ }^{4} g_{1}{ }^{2}-12 h_{1} f_{2}{ }^{4} g_{1}+\left(-2 p_{1}{ }^{2}+2 h_{0}{ }^{2}+2 h_{0} h_{1}\right. \\
& \left.+5 h_{1}{ }^{2}\right) f_{2}{ }^{4}+8{f_{2}}^{2} g_{1}{ }^{2}-12 h_{1}{f_{2}}^{2} g_{1}+\left(-p_{1}{ }^{2}+h_{0}{ }^{2}-2 h_{0} h_{1}+h_{1}{ }^{2}\right) f_{2}{ }^{2} \\
& +4 g_{1}^{2}=0
\end{aligned}
$$




$$
\begin{aligned}
& S_{2}:=\left(4 h_{0}{ }^{2}+8 h_{0} h_{1}+4 h_{1}{ }^{2}-4{p_{2}}^{2}\right) f_{1}^{4}{f_{2}}^{2}+16 f_{1}^{4} g_{1}{ }^{2}+16 h_{1} f_{1}^{4} g_{1}+4 h_{1}{ }^{2} f_{1}{ }^{4} \\
& +\left(16 \sqrt{3} h_{0} h_{1}+16 \sqrt{3} h_{1}{ }^{2}\right) f_{1}{ }^{3} f_{2}{ }^{3}+16 \sqrt{3} f_{1}{ }^{3} f_{2} g_{1} h_{1}+8 \sqrt{3} f_{1}{ }^{3} f_{2} h_{1}{ }^{2} \\
& +\left(8 h_{0}{ }^{2}+40 h_{1}{ }^{2}-8{p_{2}}^{2}\right) f_{1}{ }^{2} f_{2}{ }^{4}+32{f_{1}}^{2}{f_{2}}^{2} g_{1}{ }^{2}+16 h_{1} f_{1}{ }^{2} f_{2}{ }^{2} g_{1}+\left(8 h_{0}{ }^{2}\right. \\
& \left.+4 h_{1}{ }^{2}-8 p_{2}{ }^{2}\right) f_{1}{ }^{2}{f_{2}}^{2}+32{f_{1}}^{2} g_{1}{ }^{2}+16 h_{1} f_{1}^{2} g_{1}+\left(16 \sqrt{3} h_{0} h_{1}\right. \\
& \left.-16 \sqrt{3} h_{1}{ }^{2}\right) f_{1} f_{2}{ }^{5}+16 \sqrt{3} f_{1} f_{2}{ }^{3} g_{1} h_{1}+\left(16 \sqrt{3} h_{0} h_{1}-16 \sqrt{3} h_{1}{ }^{2}\right) f_{1} f_{2}{ }^{3} \\
& +16 \sqrt{3} f_{1} f_{2} g_{1} h_{1}+\left(4 h_{0}{ }^{2}-8 h_{0} h_{1}+4 h_{1}{ }^{2}-4{p_{2}}^{2}\right) f_{2}{ }^{6}+16{f_{2}}^{4} g_{1}{ }^{2} \\
& +\left(8 h_{0}^{2}-16 h_{0} h_{1}+8{h_{1}}^{2}-8{p_{2}}^{2}\right) f_{2}^{4}+32{f_{2}}^{2} g_{1}{ }^{2}+\left(4 h_{0}^{2}-8 h_{0} h_{1}\right. \\
& \left.+4 h_{1}{ }^{2}-4 p_{2}{ }^{2}\right) f_{2}{ }^{2}+16 g_{1}{ }^{2}=0 \\
& S_{3}:=\left(4 h_{0}{ }^{2}+8 h_{0} h_{1}+4 h_{1}^{2}-4{p_{3}}^{2}\right) f_{1}^{4}{f_{2}}^{2}+16 f_{1}^{4} g_{1}{ }^{2}+16 h_{1} f_{1}^{4} g_{1}+4 h_{1}{ }^{2} f_{1}{ }^{4} \\
& +\left(-16 \sqrt{3} h_{0} h_{1}-16 \sqrt{3} h_{1}^{2}\right) f_{1}^{3} f_{2}{ }^{3}-16 \sqrt{3} f_{1}{ }^{3} f_{2} g_{1} h_{1}-8 \sqrt{3} f_{1}{ }^{3} f_{2} h_{1}{ }^{2} \\
& +\left(8 h_{0}{ }^{2}+40 h_{1}{ }^{2}-8 p_{3}{ }^{2}\right) f_{1}{ }^{2} f_{2}{ }^{4}+32{f_{1}}^{2}{f_{2}}^{2} g_{1}{ }^{2}+16 h_{1} f_{1}{ }^{2} f_{2}{ }^{2} g_{1}+\left(8 h_{0}{ }^{2}\right. \\
& \left.+4 h_{1}^{2}-8 p_{3}^{2}\right) f_{1}^{2}{f_{2}}^{2}+32 f_{1}^{2} g_{1}^{2}+16 h_{1} f_{1}^{2} g_{1}+\left(-16 \sqrt{3} h_{0} h_{1}\right. \\
& \left.+16 \sqrt{3} h_{1}{ }^{2}\right) f_{1} f_{2}{ }^{5}-16 \sqrt{3} f_{1} f_{2}{ }^{3} g_{1} h_{1}+\left(-16 \sqrt{3} h_{0} h_{1}+16 \sqrt{3} h_{1}{ }^{2}\right) f_{1} f_{2}{ }^{3} \\
& -16 \sqrt{3} f_{1} f_{2} g_{1} h_{1}+\left(4 h_{0}{ }^{2}-8 h_{0} h_{1}+4 h_{1}{ }^{2}-4{p_{3}}^{2}\right) f_{2}{ }^{6}+16{f_{2}}^{4} g_{1}{ }^{2} \\
& +\left(8 h_{0}{ }^{2}-16 h_{0} h_{1}+8 h_{1}{ }^{2}-8{p_{3}}^{2}\right) f_{2}{ }^{4}+32{f_{2}}^{2} g_{1}{ }^{2}+\left(4 h_{0}{ }^{2}-8 h_{0} h_{1}\right. \\
& \left.+4 h_{1}{ }^{2}-4 p_{3}{ }^{2}\right) f_{2}{ }^{2}+16 g_{1}{ }^{2}=0
\end{aligned}
$$

$$
\begin{aligned}
& \left\|{ }^{0} \mathbf{r}_{C_{i}}-{ }^{0} \mathbf{r}_{B_{i}}\right\|^{2}=q_{i}^{2} \quad i=1,2,3 \Longrightarrow \\
& S_{4}:=\left(4 h_{1}^{2}-4 h_{1} h_{2}+h_{2}^{2}-q_{1}^{2}\right) c_{1}{ }^{4} c_{2}{ }^{2}+4 c_{1}{ }^{4} d_{1}{ }^{2}-4 c_{1}^{4} d_{1} h_{1}+c_{1}{ }^{4} h_{1}{ }^{2} \\
& +\left(-8 h_{1}^{2}+2 h_{2}^{2}-2 q_{1}^{2}\right) c_{1}{ }^{2} c_{2}{ }^{4}+8 c_{1}^{2} c_{2}^{2} d_{1}^{2}+8 c_{1}^{2} c_{2}^{2} d_{1} h_{1}+\left(-2 h_{1}{ }^{2}\right. \\
& \left.-6 h_{1} h_{2}+2 h_{2}{ }^{2}-2 q_{1}^{2}\right) c_{1}^{2} c_{2}^{2}+8 c_{1}^{2} d_{1}^{2}-4 c_{1}^{2} d_{1} h_{1}+\left(4 h_{1}^{2}+4 h_{1} h_{2}\right. \\
& \left.+h_{2}{ }^{2}-q_{1}{ }^{2}\right) c_{2}{ }^{6}+4 c_{2}{ }^{4} d_{1}{ }^{2}+12 c_{2}{ }^{4} d_{1} h_{1}+\left(5 h_{1}{ }^{2}+2 h_{1} h_{2}+2 h_{2}{ }^{2}\right. \\
& \left.-2 q_{1}^{2}\right) c_{2}{ }^{4}+8 c_{2}{ }^{2} d_{1}{ }^{2}+12 c_{2}{ }^{2} d_{1} h_{1}+\left(h_{1}{ }^{2}-2 h_{1} h_{2}+h_{2}{ }^{2}-q_{1}{ }^{2}\right) c_{2}{ }^{2} \\
& +4 d_{1}^{2}=0 \\
& S_{5}:=\left(h_{1}{ }^{2}+2 h_{1} h_{2}+h_{2}{ }^{2}-q_{2}{ }^{2}\right) c_{1}{ }^{4} c_{2}{ }^{2}+4 c_{1}{ }^{4} d_{1}{ }^{2}-4 c_{1}{ }^{4} d_{1} h_{1}+c_{1}{ }^{4} h_{1}{ }^{2} \\
& +\left(4 \sqrt{3} h_{1}^{2}+4 \sqrt{3} h_{1} h_{2}\right) c_{1}^{3} c_{2}^{3}-4 \sqrt{3} c_{1}{ }^{3} c_{2} d_{1} h_{1}+2 \sqrt{3} c_{1}{ }^{3} c_{2} h_{1}{ }^{2} \\
& +\left(10 h_{1}{ }^{2}+2 h_{2}{ }^{2}-2{q_{2}}^{2}\right) c_{1}{ }^{2} c_{2}{ }^{4}+8 c_{1}{ }^{2} c_{2}{ }^{2} d_{1}{ }^{2}-4 c_{1}{ }^{2} c_{2}{ }^{2} d_{1} h_{1}+\left(h_{1}{ }^{2}\right. \\
& \left.+2 h_{2}^{2}-2 q_{2}^{2}\right) c_{1}{ }^{2} c_{2}{ }^{2}+8 c_{1}^{2} d_{1}^{2}-4 c_{1}^{2} d_{1} h_{1}+\left(-4 \sqrt{3} h_{1}{ }^{2}\right. \\
& \left.+4 \sqrt{3} h_{1} h_{2}\right) c_{1} c_{2}{ }^{5}-4 \sqrt{3} c_{1} c_{2}{ }^{3} d_{1} h_{1}+\left(-4 \sqrt{3} h_{1}{ }^{2}+4 \sqrt{3} h_{1} h_{2}\right) c_{1} c_{2}{ }^{3} \\
& -4 \sqrt{3} c_{1} c_{2} d_{1} h_{1}+\left(h_{1}{ }^{2}-2 h_{1} h_{2}+h_{2}{ }^{2}-q_{2}{ }^{2}\right) c_{2}{ }^{6}+4 c_{2}{ }^{4} d_{1}{ }^{2}+\left(2 h_{1}{ }^{2}\right.
\end{aligned}
$$




$$
\begin{aligned}
& \left.-4 h_{1} h_{2}+2 h_{2}{ }^{2}-2{q_{2}}^{2}\right) c_{2}{ }^{4}+8 c_{2}{ }^{2} d_{1}{ }^{2}+\left(h_{1}{ }^{2}-2 h_{1} h_{2}+h_{2}{ }^{2}-q_{2}{ }^{2}\right) c_{2}{ }^{2} \\
& +4 d_{1}^{2}=0 \\
& S_{6}:=\left(-h_{1}{ }^{2}-2 h_{1} h_{2}-h_{2}{ }^{2}+q_{3}{ }^{2}\right) c_{1}{ }^{4} c_{2}{ }^{2}-4 c_{1}{ }^{4} d_{1}{ }^{2}+4 c_{1}{ }^{4} d_{1} h_{1}-c_{1}{ }^{4} h_{1}{ }^{2} \\
& +\left(4 \sqrt{3} h_{1}^{2}+4 \sqrt{3} h_{1} h_{2}\right) c_{1}^{3} c_{2}^{3}-4 \sqrt{3} c_{1}^{3} c_{2} d_{1} h_{1}+2 \sqrt{3} c_{1}{ }^{3} c_{2} h_{1}{ }^{2} \\
& +\left(-10 h_{1}{ }^{2}-2 h_{2}{ }^{2}+2 q_{3}{ }^{2}\right) c_{1}{ }^{2} c_{2}{ }^{4}-8 c_{1}{ }^{2} c_{2}{ }^{2} d_{1}{ }^{2}+4 c_{1}{ }^{2} c_{2}{ }^{2} d_{1} h_{1}+\left(-h_{1}{ }^{2}\right. \\
& \left.-2 h_{2}{ }^{2}+2 q_{3}{ }^{2}\right) c_{1}{ }^{2} c_{2}{ }^{2}-8 c_{1}{ }^{2} d_{1}{ }^{2}+4 c_{1}{ }^{2} d_{1} h_{1}+\left(-4 \sqrt{3} h_{1}{ }^{2}\right. \\
& \left.+4 \sqrt{3} h_{1} h_{2}\right) c_{1} c_{2}{ }^{5}-4 \sqrt{3} c_{1} c_{2}^{3} d_{1} h_{1}+\left(-4 \sqrt{3} h_{1}^{2}+4 \sqrt{3} h_{1} h_{2}\right) c_{1} c_{2}{ }^{3} \\
& -4 \sqrt{3} c_{1} c_{2} d_{1} h_{1}+\left(-h_{1}{ }^{2}+2 h_{1} h_{2}-h_{2}{ }^{2}+q_{3}{ }^{2}\right) c_{2}{ }^{6}-4 c_{2}{ }^{4} d_{1}{ }^{2}+\left(-2 h_{1}{ }^{2}\right. \\
& \left.+4 h_{1} h_{2}-2 h_{2}{ }^{2}+2 q_{3}{ }^{2}\right) c_{2}{ }^{4}-8 c_{2}{ }^{2} d_{1}{ }^{2}+\left(-h_{1}{ }^{2}+2 h_{1} h_{2}-h_{2}{ }^{2}+q_{3}{ }^{2}\right) c_{2}{ }^{2} \\
& -4 d_{1}^{2}=0
\end{aligned}
$$

By substituting the prismatic joint lengths $p_{i}$ and $q_{i}$, Eqs. (50) to (55) can be solved for the parameters $f_{1}, f_{2}, g_{1}, c_{1}, c_{2}$ and $d_{1}$. Since each module can have up to eight direct kinematics solutions ${ }^{21,24}$ in each operation mode, the 3-RPS-3-SPR S-PM can have up to 64 solutions for its direct kinematics problem in each case of Eq. (47). The transformation matrix between the fixed frame $\mathcal{F}_{0}$ and the moving platform frame $\mathcal{F}_{2}$ is established as $\mathbf{T}$ in Sec. 3. By expressing this matrix in dual quaternion form or mapping it to a point in $\mathbb{P}^{7}$ leads to the representation of the S-PM at hand in terms of the orientation Study parameters $x_{i}, i=0,1,2,3$ as follows:

$$
\left[\begin{array}{l}
x_{0} \\
x_{1} \\
x_{2} \\
x_{3}
\end{array}\right]=\left[\begin{array}{c}
c_{1} f_{1}+c_{2} f_{2}-1 \\
-c_{1}-f_{1} \\
-c_{2}-f_{2} \\
c_{1} f_{2}-c_{2} f_{1}
\end{array}\right]
$$

The expressions for the translational Study parameters $y_{i}, i=0,1,2,3$ as a function of $f_{1}, f_{2}, g_{1}, c_{1}, c_{2}$ and $d_{1}$ are shown in the Appendix. Note that every term in the right-hand side of Eq. (56) is divided by $c_{1} f_{1}+c_{2} f_{2}-1 \neq 0$. The case $c_{1} f_{1}+c_{2} f_{2}-1=0$ is a particular singularity condition identified as the case vi. a. in Table 1. It is easy to verify from Eqs. (56) and (68) that the Study parameters $x_{i}, y_{i}, i=0,1,2,3$ satisfy the Study's quadric equation:

$$
x_{0} y_{0}+x_{1} y_{1}+x_{2} y_{2}+x_{3} y_{3}=0
$$

The Plücker coordinates $\left(p_{01}, p_{02}, p_{03}, p_{23}, p_{31}, p_{12}\right)$ of the corresponding Finite Screw Axis (FSA) are given by ${ }^{35}$

$$
\begin{array}{ll}
p_{01}=\left(-x_{1}^{2}-x_{2}^{2}-x_{3}^{2}\right) x_{1}, & p_{23}=x_{0} y_{0} x_{1}-\left(-x_{1}^{2}-x_{2}^{2}-x_{3}^{2}\right) y_{1} \\
p_{02}=\left(-x_{1}^{2}-x_{2}^{2}-x_{3}^{2}\right) x_{2}, & p_{31}=x_{0} y_{0} x_{2}-\left(-x_{1}^{2}-x_{2}^{2}-x_{3}^{2}\right) y_{2} \\
p_{03}=\left(-x_{1}^{2}-x_{2}^{2}-x_{3}^{2}\right) x_{3}, & p_{12}=x_{0} y_{0} x_{3}-\left(-x_{1}^{2}-x_{2}^{2}-x_{3}^{2}\right) y_{3}
\end{array}
$$


The following conclusions are drawn from Eqs. (56) and (58):

- $x_{0}=0$ implies that the transformation is a finite screw motion ${ }^{21}$ with an angle of $180^{\circ}$, also called as a $\pi$ screw by Study. It corresponds to the singularity condition vi. in Tab. 1.

- $x_{1}=x_{2}=0$ makes $p_{01}=p_{02}=0$, implying that the direction of the FSA is vertical. It corresponds to the singularity condition iv. in Tab. 1 where the platform and the base are parallel to each other.

- $x_{3}=0$ makes $p_{03}=0$, implying that the FSA is parallel to the $x_{0} y_{0}$ plane. In this case, $c_{1} f_{2}=c_{2} f_{1}$. If $c_{1}=0$, then it corresponds to the singular configuration i. in Tab. 1.

In fact, the direct kinematics problem is solved by first calculating the parameters $f_{1}, f_{2}, g_{1}, c_{1}, c_{2}, d_{1}$ and then finding the Study parameters $x_{i}, y_{i}$, $i=0,1,2,3$ of the whole series-parallel manipulator. To derive six equations in input prismatic joint lengths $p_{j}, q_{j}, j=1,2,3$ and output Study parameters $x_{i}, y_{i}, i=0,1,2,3$ is algebraically cumbersome and is still an open problem.

\section{Inverse Kinematics Model (IKM)}

The inverse kinematics problem of the manipulator under study aims at finding the prismatic joint lengths as a function of the moving platform pose. Given the Study parameters, $x_{i}, y_{i}, i=0,1,2,3$ representing the transformation between the moving platform and the fixed base, the prismatic joint lengths $p_{j}, q_{j}, j=1,2,3$ must be determined. In other words, given points $C_{i}$ and $A_{i}$, point $B_{i}, i=1,2,3$ (refer Fig. 1) must be determined.

Let the given transformation matrix between the fixed frame $\mathcal{F}_{0}$ and the moving platform frame $\mathcal{F}_{2}$ be $\mathbf{M}$. Thus, the coordinates of point $C_{i}$ expressed in $\mathcal{F}_{2}$ as shown in Eq. (18) can be represented in $\mathcal{F}_{0}$ as ${ }^{0} \mathbf{r}_{C_{i}}=\mathbf{M}^{2} \mathbf{r}_{C_{i}}$. Let the homogeneous coordinates of point $B_{i}$ expressed in coordinate frame $\mathcal{F}_{0}$ be ${ }^{0} \mathbf{r}_{B_{i}}=\left[1, B x_{i}, B y_{i}, B z_{i}\right]$. The homogeneous coordinates of planes $\alpha_{i}$ and $\beta_{i}$ shown in Fig. 1 are:

$$
\begin{aligned}
& \alpha_{i}:\left[-\mathbf{r}_{A_{i}}^{T} \mathbf{u}_{i}, \mathbf{u}_{i}^{T}\right] \\
& \beta_{i}:\left[-\mathbf{r}_{C_{i}}^{T} \mathbf{v}_{i}, \mathbf{v}_{i}^{T}\right]
\end{aligned}
$$

where all the vectors are expressed in frame $\mathcal{F}_{0}$. To determine the points $B_{i}$, the constraints to be respected are: point $B_{i}$ must lie in the plane $\alpha_{i}$ and $\beta_{i}$ simultaneously and the distance between points $B_{i}$ and $B_{j}, i \neq j=1,2,3$ 
must be equal to the side length of the coupler triangular platform, $\sqrt{3} h_{1}$, $h_{1}$ being the circum-radius. Three constraints in each leg lead to a total of nine algebraic constraint equations:

Point $B_{i}$ belongs to plane $\alpha_{i}$ :

$$
\begin{aligned}
& F_{1}:=B y_{1}=0 \\
& F_{2}:=-\sqrt{3} B x_{2}-B y_{2} \\
& F_{3}:=\sqrt{3} B x_{3}-B y_{3}
\end{aligned}
$$

Point $B_{i}$ belongs to plane $\beta_{i}$ :

$$
\begin{aligned}
& F_{4}:=\left(-2 x_{0} x_{3}+2 x_{1} x_{2}\right) B x_{1}+\left(x_{0}^{2}-x_{1}^{2}+x_{2}^{2}-x_{3}^{2}\right) B y_{1}+\left(2 x_{0} x_{1}+\right. \\
& \left.2 x_{2} x_{3}\right) B z_{1}+2 x_{0} y_{2}+2 x_{1} y_{3}-2 x_{2} y_{0}-2 x_{3} y_{1}=0 \\
& F_{5}:=\left(-\sqrt{3} x_{0}{ }^{2}-\sqrt{3} x_{1}{ }^{2}+\sqrt{3} x_{2}{ }^{2}+\sqrt{3} x_{3}{ }^{2}+2 x_{0} x_{3}-2 x_{1} x_{2}\right) B x_{2}+\left(-2 \sqrt{3} x_{0} x_{3}\right. \\
& \left.-2 \sqrt{3} x_{1} x_{2}-x_{0}{ }^{2}+x_{1}{ }^{2}-x_{2}{ }^{2}+x_{3}{ }^{2}\right) B y_{2}+\left(2 \sqrt{3} x_{0} x_{2}-2 \sqrt{3} x_{1} x_{3}-2 x_{0} x_{1}\right. \\
& \left.-2 x_{2} x_{3}\right) B z_{2}-2 \sqrt{3} x_{0} y_{1}+2 \sqrt{3} x_{1} y_{0}+2 \sqrt{3} x_{2} y_{3}-2 \sqrt{3} x_{3} y_{2}-2 x_{0} y_{2}-2 x_{1} y_{3} \\
& +2 x_{2} y_{0}+2 x_{3} y_{1}=0 \\
& F_{6}:=\left(\sqrt{3} x_{0}{ }^{2}+\sqrt{3} x_{1}{ }^{2}-\sqrt{3} x_{2}{ }^{2}-\sqrt{3} x_{3}{ }^{2}+2 x_{0} x_{3}-2 x_{1} x_{2}\right) B x_{3}+\left(2 \sqrt{3} x_{0} x_{3}\right. \\
& \left.+2 \sqrt{3} x_{1} x_{2}-x_{0}{ }^{2}+x_{1}{ }^{2}-x_{2}{ }^{2}+x_{3}{ }^{2}\right) B y_{3}+\left(-2 \sqrt{3} x_{0} x_{2}+2 \sqrt{3} x_{1} x_{3}-2 x_{0} x_{1}\right. \\
& \left.-2 x_{2} x_{3}\right) B z_{3}+2 \sqrt{3} x_{0} y_{1}-2 \sqrt{3} x_{1} y_{0}-2 \sqrt{3} x_{2} y_{3}+2 \sqrt{3} x_{3} y_{2}-2 x_{0} y_{2}-2 x_{1} y_{3} \\
& +2 x_{2} y_{0}+2 x_{3} y_{1} \\
& \left\|\mathbf{r}_{B_{i}}-\mathbf{r}_{B_{j}}\right\|^{2}=3 h_{1}^{2} \quad i \neq j=1,2,3 \Longrightarrow \\
& F_{7}:=\left(B x_{1}-B x_{2}\right)^{2}+\left(B y_{1}-B y_{2}\right)^{2}+\left(B z_{1}-B z_{2}\right)^{2}-3 h_{1}^{2}=0 \\
& F_{8}:=\left(B x_{1}-B x_{3}\right)^{2}+\left(B y_{1}-B y_{3}\right)^{2}+\left(B z_{1}-B z_{3}\right)^{2}-3 h_{1}^{2}=0 \\
& F_{9}:=\left(B x_{2}-B x_{3}\right)^{2}+\left(B y_{2}-B y_{3}\right)^{2}+\left(B z_{2}-B z_{3}\right)^{2}-3 h_{1}^{2}=0
\end{aligned}
$$

$F_{i}, i=1, \ldots, 9$ can be solved for the nine parameters $B x_{j}, B y_{j}$ and $B z_{j}$, $j=1,2,3$ to further obtain the prismatic joint lengths.

After substituting the Study parameters and the design parameters, a Groebner basis of the constraint polynomials can be obtained over the ring $\mathbb{C}\left[h_{0}, h_{1}, h_{2}\right]$ as a function of $B x_{j}, B y_{j}$ and $B z_{j}, j=1,2,3$. A graded reverse lexicographic ordering of these variables results in a univariate polynomial of degree eight in any variable $B x_{j}, B y_{j}$ or $B z_{j}$. It shows that the inverse kinematics problem of the S-PM at hand has a maximum number of eight solutions. This is not surprising because the problem can be considered as placing three points $B_{i}$ on three skew lines $L_{i}, i=1,2,3$, where $L_{i}$ is the line of intersection of planes $\alpha_{i}$ and $\beta_{i}$ shown in Fig. 1. This is a classical geometrical problem 
Table 2: Solutions to inverse kinematics of the 3-RPS-3-SPR S-PM when $h_{0}=2, h_{1}=1, h_{2}=2$ and $\left(x_{0}: x_{1}: x_{2}: x_{3}: y_{0}: y_{1}: y_{2}: y_{3}\right)=(2.8215:$ $-1.2912:-0.3348: 1.2434: 2.1837: 1.1542: 1.6012:-3.3256)$.

\begin{tabular}{cccccccccc}
\hline & & & & & & & & & \\
\hline IKM sol. & $B x_{1}$ & $B y_{1}$ & $B z_{1}$ & $B x_{2}$ & $B y_{2}$ & $B z_{2}$ & $B y_{3}$ & $B z_{3}$ \\
1 & 1.190 & 0.0 & 1.095 & 0.922 & -1.597 & 1.710 & -0.398 & -0.689 & 1.051 \\
2 & -0.385 & 0.0 & 2.289 & -0.049 & 0.084 & 3.986 & -0.773 & -1.339 & 3.317 \\
3 & -0.867 & 0.0 & 2.650 & 0.562 & -0.972 & 2.550 & -0.412 & -0.712 & 1.140 \\
4 & -1.500 & 0.0 & 3.130 & 0.169 & -0.293 & 3.470 & -0.564 & -0.976 & 2.060 \\
5 & 0.893 & 0.0 & 1.320 & 0.991 & -1.710 & 1.540 & -0.517 & -0.895 & 1.780 \\
6 & -1.080 & 0.0 & 2.810 & 0.461 & -0.797 & 2.790 & -0.841 & -1.450 & 3.720 \\
7 & 1.210 & 0.0 & 1.080 & 0.724 & -1.250 & 2.170 & -0.384 & -0.665 & 0.975 \\
8 & -1.600 & 0.0 & 3.210 & -0.032 & 0.055 & 3.940 & -0.845 & -1.460 & 3.750 \\
\hline
\end{tabular}

and it has been proven ${ }^{36}$ that the maximum number of solutions is indeed eight with a minimal octic univariate polynomial. The interesting feature of the inverse kinematics of the 3-RPS-3-SPR S-PM is that the univariate polynomial factors into four quadratic polynomials. Further examination reveals that the four factors belong to four different combinations of the operation modes of each module like the four cases shown in Eq. (47). Since the transition between two operation modes is a constraint singularity, one of the singularities separating the IKM solutions is a constraint singularity ${ }^{21}$ in each module.

The IKM is solved for an example with Study parameters: $\left(x_{0}: x_{1}: x_{2}\right.$ : $\left.x_{3}: y_{0}: y_{1}: y_{2}: y_{3}\right)=(2.8215:-1.2912:-0.3348: 1.2434: 2.1837: 1.1542:$ $1.6012:-3.3256)$. The design parameters are chosen to be $h_{0}=2, h_{1}=$ $1, h_{2}=2$. Eight real solutions to IKM are found as shown in Table 2.

The corresponding configurations of the S-PM are displayed in Fig. 8. Moreover, the operation mode $(\mathrm{OM})$ of each module is mentioned by recalling the cases from Eq. (47):

- Case a. (IKM solutions 1 and 2 in Table 2) $c_{3}=f_{3}=0 \Longrightarrow 3$-RPS $O M 1-$ $3-\mathrm{SPR}_{O M 1}$

- Case b. (IKM solutions 3 and 4 in Table 2) $c_{0}=f_{0}=0 \Longrightarrow 3$-RPS ${ }_{O M 2}-$ $3-\mathrm{SPR}_{O M 2}$

- Case c. (IKM solutions 5 and 6 in Table 2) $c_{0}=f_{3}=0 \Longrightarrow 3-\mathrm{RPS}_{O M 2}-$ $3-\mathrm{SPR}_{O M 1}$ 
- Case d. (IKM solutions 7 and 8 in Table 2) $c_{3}=f_{0}=0 \Longrightarrow 3$-RPS ${ }_{O M 1}-$ $3-\mathrm{SPR}_{O M 2}$

There are two inverse kinematic solutions in each of these cases. How these two solutions are separated is still an open issue and is the subject of future work. Figure 9 presents the eight solutions to the inverse kinematics problem of the manipulator as eight possibilities to locate the three points, $B_{i}$ on three skew lines $L_{i}: \alpha_{i} \wedge \beta_{i}, i=1,2,3$.

\section{Conclusions}

Study parametrization of individual modules of the 3-RPS-3-SPR SeriesParallel Manipulator (S-PM) was used to determine six parameters that characterize the manipulator. The kinematic Jacobian matrix was derived and can be used to numerically determine whether a manipulator configuration is singular or not. Moreover, the serial singularities that arise due to the stacking of the two parallel modules were enumerated by mapping these singularities to the degeneracy of the characteristic tetrahedron of the S-PM. Both geometric conditions and algebraic expressions for the serial singularities were established and listed in Table 1. The Direct Kinematics Model (DKM) of the 3-RPS-3-SPR S-PM was solved to find out that the maximum number of solutions to the DKM was the product of the maximum number of solutions to the DKM of each module. When each module is restricted to lie in one of the operation modes, the maximum number of assembly modes is up to 64. Furthermore, the Inverse Kinematics Model (IKM) was solved to find out that the univariate polynomial splits into four factors based on the operation mode in which each module lies. The number of solutions to the IKM was found to be up to eight and an example was shown to depict those eight solutions.

As a part of the future work, the constraint equations will be written only as a function of input and output parameters in order to solve the DKM directly instead of splitting it into two stages as shown in this paper. Moreover, the workspace of the S-PM will be plotted and the singularities separating the solutions to IKM will be explored.

\section{Acknowledgements}

This work was conducted with the support of both Centrale Nantes and the French National Research Agency (ANR project "Kapamat" \#ANR-14CE34-0008-01). 

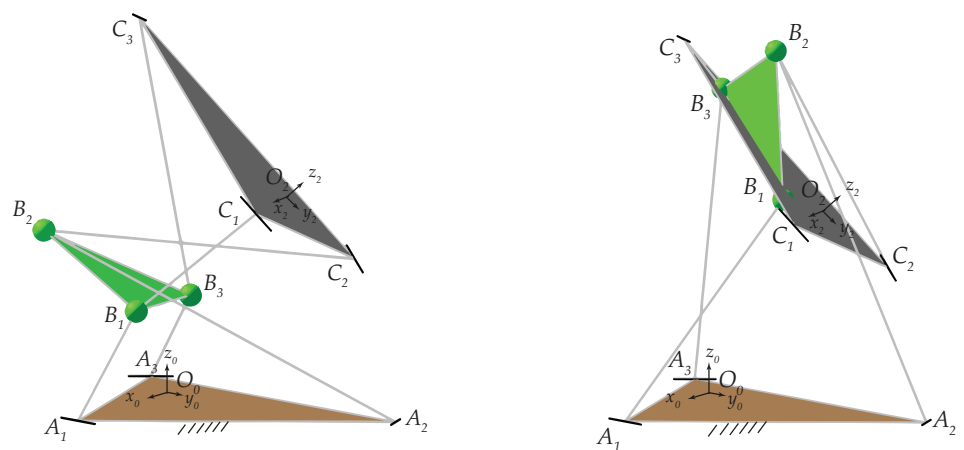

(a) Case a.
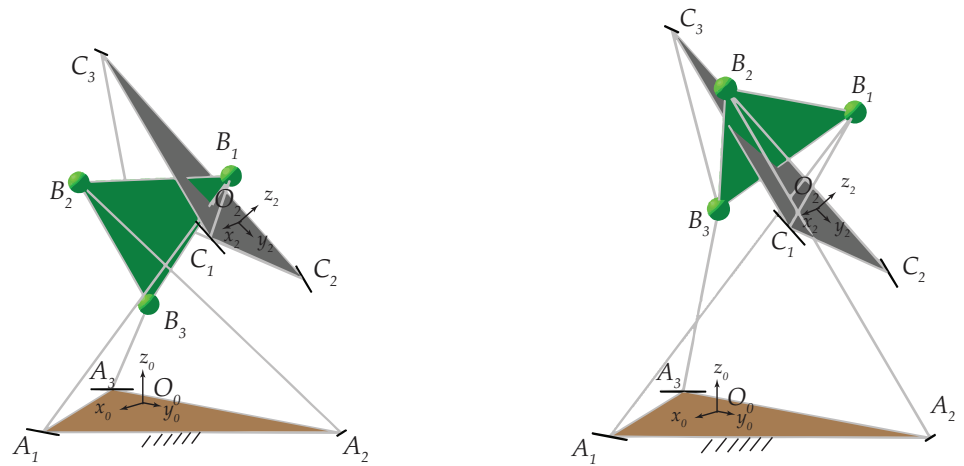

(b) Case b.
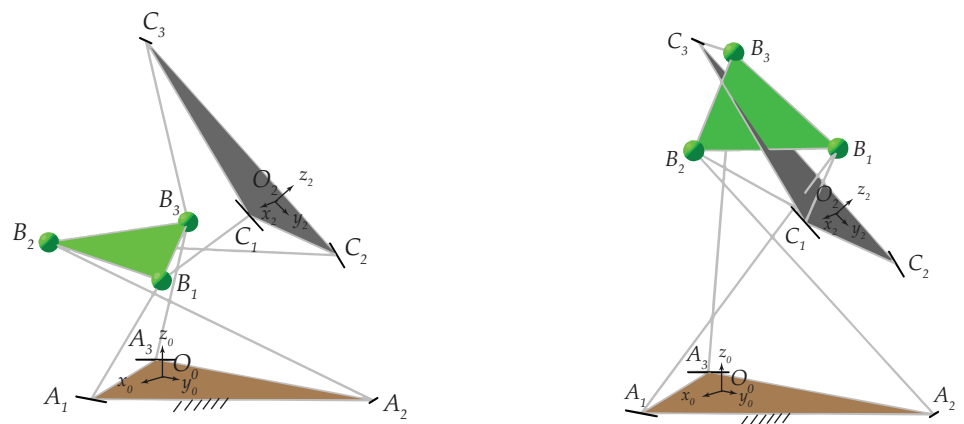

(c) Case c.
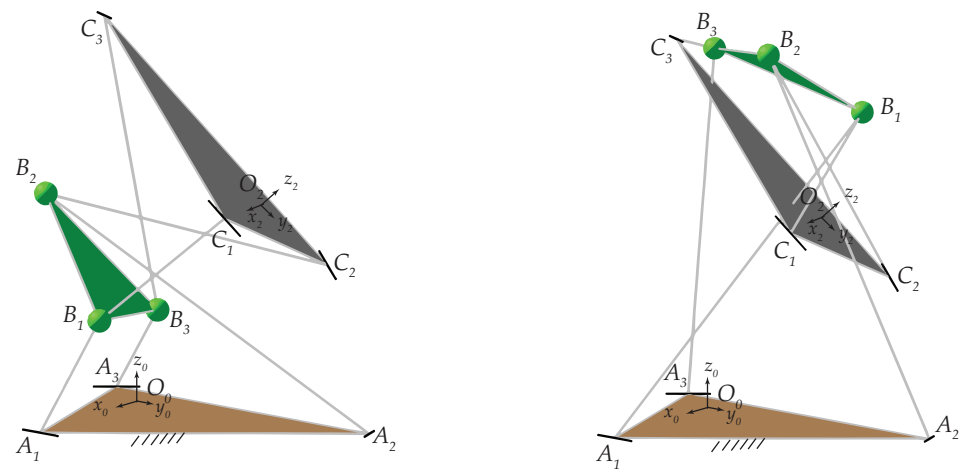

(d) Case d.

Figure 8: Eight inverse kinematic solıbtions for the 3-RPS-3-SPR S-PM when $h_{0}=2, h_{1}=1, h_{2}=2$ and $\left(x_{0}: x_{1}: x_{2}: x_{3}: y_{0}: y_{1}: y_{2}: y_{3}\right)=(2.8215:$ $-1.2912:-0.3348: 1.2434: 2.1837: 1.1542: 1.6012:-3.3256)$. 


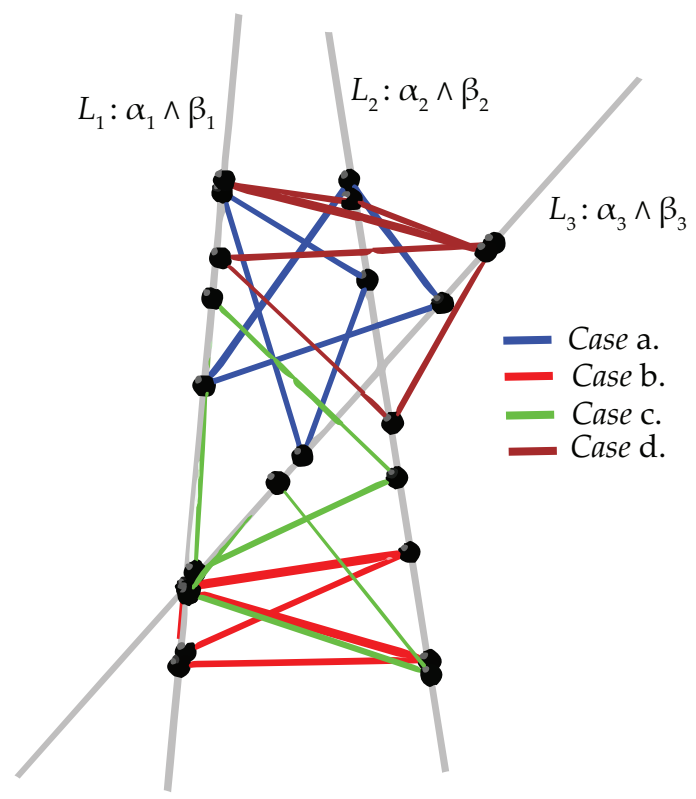

Figure 9: Eight solutions to IKM as locating 3 points on 3 lines problem

\section{References}

[1] J. P. Merlet, "Parallel robots," Springer Science \&6 Business Media (2006).

[2] L. W. Tsai, "Robot analysis: the mechanics of serial and parallel manipulators," John Wiley $\&$ Sons (1999).

[3] T. K. Tanev, "Kinematics of a hybrid (parallelserial) robot manipulator," J. Mech. Mach. Theory 35(9), 1183-1196 (2000).

[4] H. H. Cheng, J. J. Lee and R. Penkar, "Kinematic Analysis of a Hybrid Serial-and-Parallel-Driven Redundant Industrial Manipulator," Int. J. Rob. Aut. 10(4), 159-166 (1995).

[5] M. Shahinpoor, "Kinematics of a Parallel-Serial (Hybrid) Manipulator," J. Rob. Sys. 10(4), 159-166 (1995).

[6] K. J. Waldron, M. Raghavan and B. Roth, "Kinematics of a Hybrid Series-Parallel Manipulation System," J. Dyn. Sys. Measurement and Control 111(2), 211-221 (1989).

[7] C. Zhang and S-M. Song, "Geometry and Position Analysis of a Novel Class of Hybrid Manipulators," In: Proceedings of ASME Design Technical Conference (1994) pp. 1-9. 
[8] L. Romdhane, "Design and analysis of a hybrid serial-parallel manipulator," J. Mech. Mach. Theory 34(7), 1037-1055 (1999).

[9] X. Z. Zheng, H. Z. Bin, Y. G. Luo, "Kinematic analysis of a hybrid serial-parallel manipulator," Int. J. Adv. Manuf. Tech. 23(11), 925-930 (2004).

[10] B. Hu, J. Yu, Y. Lu, C. Sui and J.Han, "Statics and Stiffness Model of Serial-Parallel Manipulator Formed by k Parallel Manipulators Connected in Series," ASME. J. Mech. Rob. 4(2), pp. 021012-0210128(2012).

[11] Y. Lu and B.Hu, "Analysis of kinematics/statics and workspace of a 2(SP+SPR+SPU) serial-parallel manipulator," J. Multibody Syst. Dyn. 21(4), 361-70 (1999).

[12] J. G. Alvarado, C. R. A. Njera, L. C. Rosas, J. M. R. Martnez, M. N. Islam, "Kinematics and dynamics of 2(3-RPS) manipulators by means of screw theory and the principle of virtual work," J. Mech. Mach. Theory 43(10), 1281-1294 (2008).

[13] B. Hu and L. Yi, J. J. Yu and S. Zhuang, "Analyses of Inverse Kinematics, Statics and Workspace of a Novel 3-RPS-3-SPR Serial-Parallel Manipulator ," J. Mech. Eng. M1(6), 65-72 (2012).

[14] B. Hu, 'Formulation of unified Jacobian for serial-parallel manipulators ," Robotics and Computer-Integrated Manufacturing 30(5), 460-467 (2014).

[15] J. g. -Alvarado, L. P. -González, G. .R. Mondragón, H. R. Garduño and A. T. Villagómez, "Mobility and velocity analysis of a limited-dof seriesparallel manipulator," In: Conference : XVII COMRob 2015, Mexican Robotics Congress (2015)

[16] K. H. Hunt, "Kinematic Geometry of Mechanisms," Oxford University Press (1987).

[17] A. Nayak, S. Caro and P.Wenger, "Local and Full-Cycle Mobility Analysis of a 3-RPS-3-SPR Series-Parallel Manipulator," In: Proceedings of the 7th International Workshop on Computational Kinematics (2018) pp. 499-507.

[18] M. L. Husty, M. Pfurner, H. P. Schröcker and K. Brunnthaler, "Algebraic Methods in Mechanism Analysis and Synthesis," J. Robotica 25(6), 661675 (2007). 
[19] M. L. Husty and H. P. Schröcker, "Kinematics and Algebraic Geometry," In: 21st Century Kinematics (2013) pp. 85-123.

[20] T. Stigger and M. L. Husty, "Constraint equations of inverted kinematic chains," In: Proceedings of the 7th International Workshop on Computational Kinematics (2018) pp. 491-498.

[21] J. Schadlbauer, D. R. Walter and M. L. Husty, "The 3-RPS parallel manipulator from an algebraic viewpoint," J. Mech. Mach. Theory 75, 161-176 (2014).

[22] i. Ebert-Uphoff, J. K. Lee and H. Lipkin, "Characteristic tetrahedron of wrench singularities for parallel manipulators with three legs ," Proceedings of the Institution of Mechanical Engineers, Part C: J. Mech. Eng. Sci. 216(1), 81-93 (2002).

[23] S. A. Joshi and L. W. Tsai, "Jacobian Analysis of Limited-DOF Parallel Manipulators," ASME J. Mech. Des. 124(2), 254-258 (2002).

[24] A. Nayak, L. Nurhami, P. Wenger and S. Caro, "Comparison of 3-RPS and 3-SPR Parallel Manipulators Based on Their Maximum Inscribed Singularity-Free Circle," In: New Trends in Mechanism and Machine Science (2017) pp. 121-130.

[25] R. Kalla, L. Nurhami, S. Bandyopadhay, P. Wenger and S. Caro, "A Study of $\Sigma 2$ Singularities in the 3-RPS Parallel Manipulator," In: 2nd International and 17th National Conference on Machines and Mechanisms, India (2015)

[26] J. Schadlbauer, M. L. Husty, S. Caro and P.Wenger, "Self-motions of 3RPS manipulators," ASME J. Frontiers Mech. Eng. 8(1), 62-69 (2013).

[27] S. Amine, O. Mokhiamar and S. Caro, "Classification of 3T1R Parallel Manipulators Based on Their Wrench Graph," ASME. J. Mech. Rob. 9(1), 011003-011003-10 (2017).

[28] S. Amine, S. Caro, P. Wenger and D. Kanaan, "Singularity analysis of the H4 robot using GrassmannCayley algebra," J. Robotica 9(1), 11091118 (2012).

[29] S. Amine, M.T.Masouleh, S. Caro, P. Wenger and C.Gosselin, "Singularity conditions of 3T1R parallel manipulators with identical limb structures," ASME. J. Mech. Rob. 4(1), 011011-1011011-11 (2012). 
[30] S. Maraje, L. Nurhami, and S. Caro, "Operation Modes Comparison of a Reconfigurable 3-PRS Parallel Manipulator Based on Kinematic Performance," In: Proceedings of the ASME 2016 International Design Engineering Technical Conferences and Computers and Information in Engineering Conference, IDETC/CIE 2016, Charlotte, North Carolina, USA, August 2124. (2016)

[31] S. Caro, G. Moroz, T. Gayral, D.Chablat and C.Chen, "Singularity analysis of a six-dof parallel manipulator using grassmann-cayley algebra and groebner bases," In: In Symposium on Brain, Body and Machine, Montreal, QC., Canada, November 10-12. (2010) pp. 341-352

[32] J. P. Merlet, "Singular configurations of parallel manipulators and grassmann geometry," In: Geometry and Robotics: Workshop, Toulouse, France (1989) pp. 194-212.

[33] H. Pottmann and J. Wallner, "Computational Line Geometry," Springer-Verlag New York, Inc. (2001).

[34] i. A. Bonev, D. Zlatanov, C. M. Gosselin, "Advantages of the modified Euler angles in the design and control of PKMs," In: Parallel Kinematic Machines International Conference (2002) pp. 171-188.

[35] E. A. Weiss, "Einführung in die Liniengeometrie und Kinematik," B. G. Teubner, Leibzig (1935).

[36] P. J. Zsombor-Murray and A. Gfrerrer, "On Three-Legged Six Degree of Freedom Double Triangular Manipulator Direct Kinematics," (2009).

\section{Appendix}

Direct Kinematics: Translational parameters, $y_{i}, i=0,1,2,3$ as functions of $f_{1}, f_{2}, g_{1}, c_{1}, c_{2}, d_{1}$.

$$
\begin{aligned}
& y_{0}=\frac{1}{2\left(f_{1}{ }^{2}+f_{2}{ }^{2}+1\right)\left(c_{1}{ }^{2}+c_{2}{ }^{2}+1\right) c_{2} f_{2}}\left(2 c_{1}{ }^{3} c_{2} f_{1}{ }^{2} f_{2} g_{1}+c_{1}{ }^{3} c_{2} f_{1}{ }^{2} f_{2} h_{1}+2 c_{1}{ }^{3} c_{2} f_{2}{ }^{3} g_{1}\right. \\
& +c_{1}^{3} c_{2} f_{2}{ }^{3} h_{1}-2 c_{1}{ }^{3} d_{1} f_{1}{ }^{2} f_{2}{ }^{2}-2 c_{1}{ }^{3} d_{1} f_{2}{ }^{4}+c_{1}^{3} f_{1}{ }^{2} f_{2}{ }^{2} h_{1}+c_{1}{ }^{3} f_{2}{ }^{4} h_{1}-2 c_{1}{ }^{2} c_{2}{ }^{2} f_{1}{ }^{3} g_{1} \\
& -c_{1}^{2} c_{2}^{2} f_{1}^{3} h_{1}-2 c_{1}^{2} c_{2}^{2} f_{1} f_{2}^{2} g_{1}+3 c_{1}^{2} c_{2}^{2} f_{1} f_{2}^{2} h_{1}+2 c_{1}^{2} c_{2} d_{1} f_{1}^{3} f_{2}+2 c_{1}^{2} c_{2} d_{1} f_{1} f_{2}{ }^{3} \\
& -c_{1}^{2} c_{2} f_{1}^{3} f_{2} h_{1}+3 c_{1}^{2} c_{2} f_{1} f_{2}{ }^{3} h_{1}+2 c_{1} c_{2}^{3} f_{1}^{2} f_{2} g_{1}-3 c_{1} c_{2}{ }^{3} f_{1}^{2} f_{2} h_{1}+2 c_{1} c_{2}^{3} f_{2}^{3} g_{1} \\
& -3 c_{1} c_{2}^{3} f_{2}^{3} h_{1}-2 c_{1} c_{2}^{2} d_{1} f_{1}^{2} f_{2}^{2}-2 c_{1} c_{2}^{2} d_{1} f_{2}^{4}-3 c_{1} c_{2}^{2} f_{1}^{2} f_{2}^{2} h_{1}-3 c_{1} c_{2}{ }^{2} f_{2}{ }^{4} h_{1} \\
& -2 c_{2}{ }^{4} f_{1}^{3} g_{1}-c_{2}{ }^{4} f_{1}{ }^{3} h_{1}-2 c_{2}{ }^{4} f_{1} f_{2}{ }^{2} g_{1}+3 c_{2}{ }^{4} f_{1} f_{2}{ }^{2} h_{1}+2 c_{2}{ }^{3} d_{1} f_{1}{ }^{3} f_{2}+2 c_{2}{ }^{3} d_{1} f_{1} f_{2}{ }^{3}
\end{aligned}
$$


$-c_{2}^{3} f_{1}^{3} f_{2} h_{1}+3 c_{2}{ }^{3} f_{1} f_{2}{ }^{3} h_{1}+2 c_{1}{ }^{3} c_{2} f_{2} g_{1}+c_{1}{ }^{3} c_{2} f_{2} h_{1}-2 c_{1}{ }^{3} d_{1} f_{2}{ }^{2}+c_{1}{ }^{3} f_{2}{ }^{2} h_{1}$

$-2 c_{1}^{2} c_{2}^{2} f_{1} g_{1}+2 c_{1}^{2} c_{2} d_{1} f_{1} f_{2}+2 c_{1} c_{2}{ }^{3} f_{2} g_{1}-3 c_{1} c_{2}{ }^{3} f_{2} h_{1}-2 c_{1} c_{2}{ }^{2} d_{1} f_{2}{ }^{2}-3 c_{1} c_{2}{ }^{2} f_{2}{ }^{2} h_{1}$

$+2 c_{1} c_{2} f_{1}{ }^{2} f_{2} g_{1}+2 c_{1} c_{2} f_{2}{ }^{3} g_{1}-2 c_{1} d_{1} f_{1}{ }^{2} f_{2}{ }^{2}-2 c_{1} d_{1} f_{2}{ }^{4}-2 c_{2}{ }^{4} f_{1} g_{1}+2 c_{2}{ }^{3} d_{1} f_{1} f_{2}$

$-2 c_{2}{ }^{2} f_{1}^{3} g_{1}-c_{2}{ }^{2} f_{1}{ }^{3} h_{1}-2 c_{2}{ }^{2} f_{1} f_{2}{ }^{2} g_{1}+3 c_{2}{ }^{2} f_{1} f_{2}{ }^{2} h_{1}+2 c_{2} d_{1} f_{1}{ }^{3} f_{2}+2 c_{2} d_{1} f_{1} f_{2}{ }^{3}$

$\left.-c_{2} f_{1}^{3} f_{2} h_{1}+3 c_{2} f_{1} f_{2}{ }^{3} h_{1}+2 c_{1} c_{2} f_{2} g_{1}-2 c_{1} d_{1} f_{2}{ }^{2}-2 c_{2}{ }^{2} f_{1} g_{1}+2 c_{2} d_{1} f_{1} f_{2}\right)$

$$
\begin{aligned}
& y_{1}=\frac{1}{2\left(f_{1}{ }^{2}+f_{2}{ }^{2}+1\right)\left(c_{1}{ }^{2}+c_{2}{ }^{2}+1\right) c_{2} f_{2}}\left(4 c_{1}{ }^{3} c_{2} f_{1} f_{2}{ }^{3} h_{1}+4 c_{1}{ }^{2} c_{2}{ }^{2} f_{2}{ }^{4} h_{1}-4 c_{1} c_{2}{ }^{3} f_{1}{ }^{3} f_{2} h_{1}\right. \\
& -4 c_{2}^{4} f_{1}^{2} f_{2}^{2} h_{1}+c_{1}^{3} c_{2} f_{1} f_{2} h_{1}-2 c_{1}^{2} c_{2}^{2} f_{1}^{2} g_{1}-c_{1}^{2} c_{2}^{2} f_{1}^{2} h_{1}-2 c_{1}{ }^{2} c_{2}^{2} f_{2}^{2} g_{1} \\
& +4 c_{1}^{2} c_{2}^{2} f_{2}^{2} h_{1}-2 c_{1}^{2} c_{2} d_{1} f_{1}^{2} f_{2}-2 c_{1}^{2} c_{2} d_{1} f_{2}^{3}-2 c_{1}^{2} c_{2} f_{1}^{2} f_{2} g_{1}-2 c_{1}{ }^{2} c_{2} f_{2}{ }^{3} g_{1} \\
& -2 c_{1}^{2} d_{1} f_{1}^{2} f_{2}{ }^{2}-2 c_{1}{ }^{2} d_{1} f_{2}{ }^{4}+c_{1}{ }^{2} f_{1}{ }^{2} f_{2}{ }^{2} h_{1}+c_{1}{ }^{2} f_{2}{ }^{4} h_{1}-3 c_{1} c_{2}{ }^{3} f_{1} f_{2} h_{1}-c_{1} c_{2} f_{1}{ }^{3} f_{2} h_{1} \\
& +3 c_{1} c_{2} f_{1} f_{2}{ }^{3} h_{1}-2 c_{2}{ }^{4} f_{1}^{2} g_{1}-c_{2}{ }^{4} f_{1}{ }^{2} h_{1}-2 c_{2}{ }^{4} f_{2}{ }^{2} g_{1}-2 c_{2}{ }^{3} d_{1} f_{1}{ }^{2} f_{2}-2 c_{2}{ }^{3} d_{1} f_{2}{ }^{3} \\
& -2 c_{2}{ }^{3} f_{1}^{2} f_{2} g_{1}-2 c_{2}{ }^{3} f_{2}{ }^{3} g_{1}-2 c_{2}{ }^{2} d_{1} f_{1}{ }^{2} f_{2}{ }^{2}-2 c_{2}{ }^{2} d_{1} f_{2}{ }^{4}-4 c_{2}{ }^{2} f_{1}{ }^{2} f_{2}{ }^{2} h_{1}-2 c_{1}{ }^{2} c_{2}{ }^{2} g_{1} \\
& -2 c_{1}^{2} c_{2} d_{1} f_{2}-2 c_{1}^{2} c_{2} f_{2} g_{1}-2 c_{1}^{2} d_{1} f_{2}{ }^{2}+c_{1}^{2} f_{2}{ }^{2} h_{1}-2 c_{2}^{4} g_{1}-2 c_{2}{ }^{3} d_{1} f_{2}-2 c_{2}{ }^{3} f_{2} g_{1} \\
& -2 c_{2}^{2} d_{1} f_{2}^{2}-2 c_{2}^{2} f_{1}^{2} g_{1}-c_{2}{ }^{2} f_{1}{ }^{2} h_{1}-2 c_{2}^{2} f_{2}^{2} g_{1}-2 c_{2} d_{1} f_{1}^{2} f_{2}-2 c_{2} d_{1} f_{2}{ }^{3}-2 c_{2} f_{1}{ }^{2} f_{2} g_{1} \\
& \left.-2 c_{2} f_{2}{ }^{3} g_{1}-2 d_{1} f_{1}{ }^{2} f_{2}{ }^{2}-2 d_{1} f_{2}{ }^{4}-2 c_{2}{ }^{2} g_{1}-2 c_{2} d_{1} f_{2}-2 c_{2} f_{2} g_{1}-2 d_{1} f_{2}{ }^{2}\right)
\end{aligned}
$$

$$
\begin{aligned}
& y_{2}=\frac{1}{2\left(f_{1}{ }^{2}+f_{2}{ }^{2}+1\right)\left(c_{1}{ }^{2}+c_{2}{ }^{2}+1\right) c_{2} f_{2}}\left(4 c_{1}{ }^{3} c_{2} f_{1}{ }^{2} f_{2}{ }^{2} h_{1}-4 c_{1}{ }^{2} c_{2}{ }^{2} f_{1}{ }^{3} f_{2} h_{1}-4 c_{1} c_{2}{ }^{3} f_{2}{ }^{4} h_{1}\right. \\
& +4 c_{2}^{4} f_{1} f_{2}{ }^{3} h_{1}+2 c_{1}{ }^{3} c_{2} f_{1}{ }^{2} g_{1}+c_{1}{ }^{3} c_{2} f_{1}{ }^{2} h_{1}+2 c_{1}{ }^{3} c_{2} f_{2}{ }^{2} g_{1}+2 c_{1}{ }^{3} d_{1} f_{1}{ }^{2} f_{2}+2 c_{1}{ }^{3} d_{1} f_{2}{ }^{3} \\
& -c_{1}^{3} f_{1}^{2} f_{2} h_{1}-c_{1}{ }^{3} f_{2}^{3} h_{1}-3 c_{1}^{2} c_{2}^{2} f_{1} f_{2} h_{1}+2 c_{1}{ }^{2} c_{2} f_{1}{ }^{3} g_{1}+c_{1}{ }^{2} c_{2} f_{1}{ }^{3} h_{1}+2 c_{1}{ }^{2} c_{2} f_{1} f_{2}{ }^{2} g_{1} \\
& -3 c_{1}{ }^{2} c_{2} f_{1} f_{2}{ }^{2} h_{1}+2 c_{1}{ }^{2} d_{1} f_{1}^{3} f_{2}+2 c_{1}^{2} d_{1} f_{1} f_{2}{ }^{3}-c_{1}{ }^{2} f_{1}^{3} f_{2} h_{1}-c_{1}{ }^{2} f_{1} f_{2}{ }^{3} h_{1}+2 c_{1} c_{2}{ }^{3} f_{1}{ }^{2} g_{1} \\
& +c_{1} c_{2}{ }^{3} f_{1}{ }^{2} h_{1}+2 c_{1} c_{2}^{3} f_{2}{ }^{2} g_{1}-4 c_{1} c_{2}{ }^{3} f_{2}{ }^{2} h_{1}+2 c_{1} c_{2}{ }^{2} d_{1} f_{1}^{2} f_{2}+2 c_{1} c_{2}{ }^{2} d_{1} f_{2}{ }^{3} \\
& +3 c_{1} c_{2}^{2} f_{1}^{2} f_{2} h_{1}+3 c_{1} c_{2}{ }^{2} f_{2}{ }^{3} h_{1}+3 c_{1} c_{2} f_{1}{ }^{2} f_{2}{ }^{2} h_{1}-c_{1} c_{2} f_{2}{ }^{4} h_{1}+c_{2}{ }^{4} f_{1} f_{2} h_{1}+2 c_{2}{ }^{3} f_{1}{ }^{3} g_{1} \\
& +c_{2}{ }^{3} f_{1}{ }^{3} h_{1}+2 c_{2}{ }^{3} f_{1} f_{2}{ }^{2} g_{1}-3 c_{2}{ }^{3} f_{1} f_{2}{ }^{2} h_{1}+2 c_{2}{ }^{2} d_{1} f_{1}{ }^{3} f_{2}+2 c_{2}{ }^{2} d_{1} f_{1} f_{2}{ }^{3}+4 c_{2}{ }^{2} f_{1} f_{2}{ }^{3} h_{1} \\
& +2 c_{1}^{3} c_{2} g_{1}+2 c_{1}^{3} d_{1} f_{2}-c_{1}^{3} f_{2} h_{1}+2 c_{1}^{2} c_{2} f_{1} g_{1}+2 c_{1}^{2} d_{1} f_{1} f_{2}-c_{1}^{2} f_{1} f_{2} h_{1}+2 c_{1} c_{2}^{3} g_{1} \\
& +2 c_{1} c_{2}^{2} d_{1} f_{2}+3 c_{1} c_{2}^{2} f_{2} h_{1}+2 c_{1} c_{2} f_{1}^{2} g_{1}+c_{1} c_{2} f_{1}^{2} h_{1}+2 c_{1} c_{2} f_{2}{ }^{2} g_{1}-c_{1} c_{2} f_{2}{ }^{2} h_{1} \\
& +2 c_{1} d_{1} f_{1}^{2} f_{2}+2 c_{1} d_{1} f_{2}{ }^{3}+2 c_{2}^{3} f_{1} g_{1}+2 c_{2}{ }^{2} d_{1} f_{1} f_{2}+c_{2}{ }^{2} f_{1} f_{2} h_{1}+2 c_{2} f_{1}{ }^{3} g_{1}+c_{2} f_{1}{ }^{3} h_{1} \\
& +2 c_{2} f_{1} f_{2}{ }^{2} g_{1}-3 c_{2} f_{1} f_{2}{ }^{2} h_{1}+2 d_{1} f_{1}{ }^{3} f_{2}+2 d_{1} f_{1} f_{2}{ }^{3}+2 c_{1} c_{2} g_{1}+2 c_{1} d_{1} f_{2}+2 c_{2} f_{1} g_{1} \\
& \left.+2 d_{1} f_{1} f_{2}\right)
\end{aligned}
$$

$$
y_{3}=\frac{1}{2\left(f_{1}{ }^{2}+f_{2}{ }^{2}+1\right)\left(c_{1}{ }^{2}+c_{2}{ }^{2}+1\right) c_{2} f_{2}}\left(2 c_{1}^{3} c_{2} f_{1}{ }^{3} g_{1}+c_{1}{ }^{3} c_{2} f_{1}{ }^{3} h_{1}+2 c_{1}{ }^{3} c_{2} f_{1} f_{2}{ }^{2} g_{1}\right.
$$


$-3 c_{1}^{3} c_{2} f_{1} f_{2}{ }^{2} h_{1}-2 c_{1}{ }^{3} d_{1} f_{1}{ }^{3} f_{2}-2 c_{1}^{3} d_{1} f_{1} f_{2}{ }^{3}+c_{1}{ }^{3} f_{1}{ }^{3} f_{2} h_{1}+c_{1}{ }^{3} f_{1} f_{2}{ }^{3} h_{1}$

$+2 c_{1}{ }^{2} c_{2}^{2} f_{1}^{2} f_{2} g_{1}-3 c_{1}^{2} c_{2}^{2} f_{1}^{2} f_{2} h_{1}+2 c_{1}{ }^{2} c_{2}{ }^{2} f_{2}{ }^{3} g_{1}-3 c_{1}{ }^{2} c_{2}{ }^{2} f_{2}{ }^{3} h_{1}-2 c_{1}{ }^{2} c_{2} d_{1} f_{1}{ }^{2} f_{2}{ }^{2}$

$-2 c_{1}{ }^{2} c_{2} d_{1} f_{2}{ }^{4}-3 c_{1}{ }^{2} c_{2} f_{1}{ }^{2} f_{2}{ }^{2} h_{1}+c_{1}{ }^{2} c_{2} f_{2}{ }^{4} h_{1}+2 c_{1} c_{2}{ }^{3} f_{1}{ }^{3} g_{1}+c_{1} c_{2}{ }^{3} f_{1}{ }^{3} h_{1}$

$+2 c_{1} c_{2}^{3} f_{1} f_{2}{ }^{2} g_{1}-3 c_{1} c_{2}{ }^{3} f_{1} f_{2}{ }^{2} h_{1}-2 c_{1} c_{2}{ }^{2} d_{1} f_{1}{ }^{3} f_{2}-2 c_{1} c_{2}{ }^{2} d_{1} f_{1} f_{2}{ }^{3}-3 c_{1} c_{2}{ }^{2} f_{1}{ }^{3} f_{2} h_{1}$

$-3 c_{1} c_{2}{ }^{2} f_{1} f_{2}{ }^{3} h_{1}+2 c_{2}{ }^{4} f_{1}{ }^{2} f_{2} g_{1}+c_{2}{ }^{4} f_{1}{ }^{2} f_{2} h_{1}+2 c_{2}{ }^{4} f_{2}{ }^{3} g_{1}+c_{2}{ }^{4} f_{2}{ }^{3} h_{1}-2 c_{2}{ }^{3} d_{1} f_{1}{ }^{2} f_{2}{ }^{2}$

$-2 c_{2}{ }^{3} d_{1} f_{2}{ }^{4}-3 c_{2}{ }^{3} f_{1}{ }^{2} f_{2}{ }^{2} h_{1}+c_{2}{ }^{3} f_{2}{ }^{4} h_{1}+2 c_{1}{ }^{3} c_{2} f_{1} g_{1}-2 c_{1}{ }^{3} d_{1} f_{1} f_{2}+c_{1}{ }^{3} f_{1} f_{2} h_{1}$

$+2 c_{1}^{2} c_{2}^{2} f_{2} g_{1}-3 c_{1}^{2} c_{2}^{2} f_{2} h_{1}-2 c_{1}^{2} c_{2} d_{1} f_{2}^{2}-2 c_{1}^{2} c_{2} f_{1}^{2} g_{1}-c_{1}^{2} c_{2} f_{1}^{2} h_{1}-2 c_{1}^{2} c_{2} f_{2}{ }^{2} g_{1}$

$+c_{1}^{2} c_{2} f_{2}{ }^{2} h_{1}+2 c_{1}^{2} d_{1} f_{1}^{2} f_{2}+2 c_{1}^{2} d_{1} f_{2}{ }^{3}-c_{1}^{2} f_{1}^{2} f_{2} h_{1}-c_{1}{ }^{2} f_{2}{ }^{3} h_{1}+2 c_{1} c_{2}{ }^{3} f_{1} g_{1}$

$-2 c_{1} c_{2}^{2} d_{1} f_{1} f_{2}-3 c_{1} c_{2}{ }^{2} f_{1} f_{2} h_{1}+2 c_{1} c_{2} f_{1}^{3} g_{1}+c_{1} c_{2} f_{1}^{3} h_{1}+2 c_{1} c_{2} f_{1} f_{2}{ }^{2} g_{1}$

$-3 c_{1} c_{2} f_{1} f_{2}{ }^{2} h_{1}-2 c_{1} d_{1} f_{1}{ }^{3} f_{2}-2 c_{1} d_{1} f_{1} f_{2}{ }^{3}+2 c_{2}{ }^{4} f_{2} g_{1}+c_{2}{ }^{4} f_{2} h_{1}-2 c_{2}{ }^{3} d_{1} f_{2}{ }^{2}$

$-2 c_{2}{ }^{3} f_{1}{ }^{2} g_{1}-c_{2}{ }^{3} f_{1}{ }^{2} h_{1}-2 c_{2}{ }^{3} f_{2}{ }^{2} g_{1}+c_{2}{ }^{3} f_{2}{ }^{2} h_{1}+2 c_{2}{ }^{2} d_{1} f_{1}{ }^{2} f_{2}+2 c_{2}{ }^{2} d_{1} f_{2}{ }^{3}$

$+2 c_{2}{ }^{2} f_{1}^{2} f_{2} g_{1}+c_{2}{ }^{2} f_{1}{ }^{2} f_{2} h_{1}+2 c_{2}{ }^{2} f_{2}{ }^{3} g_{1}+c_{2}{ }^{2} f_{2}{ }^{3} h_{1}-2 c_{2} d_{1} f_{1}{ }^{2} f_{2}{ }^{2}-2 c_{2} d_{1} f_{2}{ }^{4}$

$-3 c_{2} f_{1}^{2} f_{2}^{2} h_{1}+c_{2} f_{2}^{4} h_{1}-2 c_{1}^{2} c_{2} g_{1}+2 c_{1}^{2} d_{1} f_{2}-c_{1}^{2} f_{2} h_{1}+2 c_{1} c_{2} f_{1} g_{1}-2 c_{1} d_{1} f_{1} f_{2}$

$-2 c_{2}{ }^{3} g_{1}+2 c_{2}{ }^{2} d_{1} f_{2}+2 c_{2}{ }^{2} f_{2} g_{1}+c_{2}{ }^{2} f_{2} h_{1}-2 c_{2} d_{1} f_{2}{ }^{2}-2 c_{2} f_{1}{ }^{2} g_{1}-c_{2} f_{1}{ }^{2} h_{1}-2 c_{2} f_{2}{ }^{2} g_{1}$

$\left.+c_{2} f_{2}{ }^{2} h_{1}+2 d_{1} f_{1}^{2} f_{2}+2 d_{1} f_{2}{ }^{3}-2 c_{2} g_{1}+2 d_{1} f_{2}\right)$ 